\title{
Polynomial Deformations and Cohomology of Calabi-Yau Manifolds
}

\author{
Paul Green ${ }^{1}$ and Tristan Hübsch ${ }^{2}$ \\ ${ }^{1}$ Department of Mathematics, University of Maryland, College Park, MD 20742, USA \\ ${ }^{2}$ Department of Physics and Astronomy, University of Maryland, College Park, MD 20742, USA
}

\begin{abstract}
We study the method of polynomial deformations that is used in the physics literature to determine the Hodge numbers of Calabi-Yau manifolds as well as the related Yukawa couplings. We show that the argument generally presented in the literature in support of these computations is seriously misleading, give a correct proof which applies to all the cases we found in the literature, and present examples which show that the method is not universally valid. We present a general analysis which applies to all Calabi-Yau manifolds embedded as complete intersections in products of complex projective spaces, yields sufficient conditions for the validity of the polynomial deformation method, and provides an alternative computation of all the Hodge numbers in many cases in which the polynomial method fails.
\end{abstract}

\section{Introduction}

Compact Kähler Ricci-flat (usually called Calabi-Yau) manifolds, of complex dimension $3\left(\mathscr{M}_{\mathrm{CY}}\right)$, were recently proposed $[1,2]$ for compactification of certain superstring theories [3]. In this way, one can obtain an $N=1$ locally supersymmetric grand-unified model (hereafter "effective model") in 4-dimensional Minkowski space-time, with the gauge group being a subgroup of $E_{6} \times E_{8}$ and the matter superfields coming in a chiral representation in general.

The massless superfields of the effective model can be represented by harmonic exterior forms on the internal $\mathscr{M}_{\mathrm{CY}}[1,2,4]^{1}$ and consequently the couplings in the effective model are related [6] by certain integrals of products of forms over $\mathscr{M}_{\mathrm{CY}}$, and possibly its nontrivial submanifolds (see also $[4,7]$ ). These integrals, in general, depend on the complex structure and the cohomology class of the Kähler form and are often calculable. Moreover, their relative values and, in particular, the identical vanishing of some of them can be deduced by applying the WignerEckart theorem, noting that the superfields (i.e. the corresponding forms on $\mathscr{M}_{\mathrm{CY}}$ )

\footnotetext{
${ }^{1}$ There does, however, exist a class of superfields, invariant under the Yang-Mills gauge group,
} that escapes this classification $[4,5]$, but this is irrelevant for the purpose of our present paper 
transform, in general, nontrivially under the (discrete) groups of symmetries of $\mathscr{M}_{\mathrm{CY}}$.

A method for computation of transformation properties of harmonic $(2,1)$ forms (which are of most interest for phenomenological applications) was proposed in [2] and applied [2,8] to the cases of some relatively simple $\mathscr{M}_{\mathrm{CY}}$. We shall refer to it as the Polynomial Deformation Method (PDM for short), since it is presented in the physics literature as an application of the theory of deformations of the complex structure of complex manifolds as developed by K. Kodaira and D.C. Spencer [9]. In the cases mentioned PDM seems to be quite powerful and in accord with other means of computation.

We shall show in this paper that PDM really depends not on the KodairaSpencer deformation theory but on a simple cohomology computation which is however not universally valid. We shall also establish that PDM is valid for all the cases we found in the literature $[2,8]$ (see Corollary to Theorem 2), and by presenting a more complicated analysis involving spectral sequences, which is the correct generalization of PDM to all configurations, establish some general criteria for the validity of PDM.

In Sect. 2 we describe our notation, which is an extension of the notation of $[4,10]$. We then briefly review the main steps of PDM and then analyze it from a general standpoint in Sect. 3. In Sect. 4 we review the general technique of spectral sequences. In Sects. 5 and 6 we construct the relevant spectral sequences and present the analysis which generalizes PDM and applies to all configurations. We summarize our conclusions in Sect. 7. Several simple, yet non-trivial, examples are given in Appendices A and B, to serve as a practical guide for further application of our method, and Appendix C, to illustrate some obstructions to completing the computation of the Hodge numbers.

\section{Configurations and Diagrams}

A configuration matrix is a pair consisting of an $m$-dimensional positive integer valued column vector $n$ and an $m \times h$-dimensional non-negative integer valued matrix $q$. It is intended to represent the degrees of homogeneity of $h$ homogeneous polynomial constraints defining an embedded variety of codimension $h$ in $\prod_{r=1}^{m} C P_{r}^{n_{r}}$. A configuration is defined to be an equivalence class of configurations matrices with respect to column exchange of $q$ and row exchange of $(n \| q)$.

We proved in [10] that the variety corresponding to a generic choice of constraint polynomials is a non-singular complex manifold. Each such manifold is said to belong to the configuration, which in turn is said to represent the manifold.

We call a configuration $(n \| q)$ minimal if the set of varieties belonging to it is not

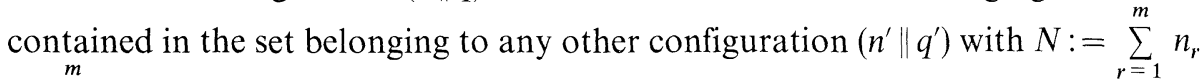
$<\sum_{r=1}^{m} n_{r}^{\prime}=: N^{\prime}$

Since we will be interested in minimal configurations representing nonnegative manifolds (i.e. manifolds with non-negative first Chern class), we require:

$$
\sum_{a=1}^{h} q_{a}^{r} \leqq n_{r}+1 \text { for all } r \text {, and } \sum_{r=1}^{m} q_{a}^{r} \geqq 2 \text { for all } a \text {. }
$$


A configuration is called decomposable if it contains a configuration of the form $(n \| q)$ with $q$ in a block-diagonal form with more than one block; otherwise it is called indecomposable.

We note that a configuration defines Calabi-Yau manifolds of dimension 3 if $N-h=3$, and $\sum_{a=1}^{h} q_{a}^{r}=n_{r}+1$ for all $r$.

We will also find it useful to introduce a diagramatic representation of the classes of embeddings represented by our configurations:

For every $C P^{n}$ we introduce a hollow circle with $n+1$ legs. For every constraint we introduce a dot. We connect some of the free ends of the legs to the dots, requiring that each dot has at least two legs connected to it.

There is an obvious 1-1 correspondence between the class of diagrams defined above and the class of configurations satisfying Rel. (1). Note that decomposable configurations correspond to disconnected diagrams. A diagram represents Calabi-Yau manifolds if and only if no legs are left with free ends. The dimension of the manifold represented by the diagram is $l-m-h$, where $l$ is the number of legs, $m$ the number of circles and $h$ the number of dots. We will also refer to this number as the dimension of the diagram. It is always non-negative since $l \geqq 2 m$ and $l \geqq 2 h$.

There is a well-known algorithm (see ref. $[4,10]$ ) for computing the Euler Character $\chi_{E}$ of the non-singular complex manifolds belonging to each configuration. We define in [10] a class of favourable configurations for which $b_{2}$, and therefore all the Hodge numbers, can be computed as well by iterating the Lefeschetz Hyperplane Theorem. The methods of the present paper will compute the Hodge numbers of the manifolds represented by many unfavourable configurations.

It will turn out to be useful to consider a special class of connected diagrams. They are characterized by the fact that they have at least one dot by deletion of which the diagram becomes disconnected. We shall refer to these diagrams as onedot-decomposable (1 DD).

Proposition 1. The complement of a decomposing dot, in a minimal diagram of dimension n, has two components which represent manifolds of positive dimension the sum of which is $n+1$.

Proof. In each component we have:

$$
l \geqq 2 m \text { and } l>2 k \Rightarrow 2 l>2(m+k) .
$$

Therefore $l-m-k>0$ for both components.

We choose to index a decomposing dot by these dimensions and refer to it as a $x-y$-dot, where $x$ and $y$ are the dimensions of the manifolds defined by the components of the complement of the decomposing dot.

A connected diagram is called 1-leg-decomposable (1 LD) if the complement of a single leg is disconnected. Clearly, every $1 \mathrm{LD}$ diagram is also $1 \mathrm{DD}$, since the decomposing leg must be connected to a dot, the removal of which disconnects the diagram as well.

The complement of a decomposing leg in a minimal diagram representing an $n$-fold has two components containing, respectively, the dot and the circle incident 
with the deleted leg. We shall refer to these respectively as the dot-complement and the circle-complement of the leg in question. We write $l_{d}, m_{d}$ and $k_{d}$ for the number of legs, circles and dots in the dot-complement, and $l_{c}, m_{c}$ and $\mathrm{k}_{c}$ for the corresponding numbers in the circle-complement. We index the leg by $l_{d}-m_{d}-k_{d}$.

Proposition 2. The possible indices for a decomposing leg in a diagram representing an $n$-fold are $0,1 \ldots n-1$.

Proof. The dot-complement of a decomposing leg, without the dot incident with the leg, is one of the two disconnected parts of the complement of this dot. By Proposition 1, it represents a manifold of dimension $1, \ldots, n$. With the dot, $l_{d}-m_{d}$ $-k_{d}$ reduces by one.

Proposition 3. A minimal configuration representing an $n$-fold has no decomposing $(n-1)$-leg.

Proof. If it had, the circle-complement of the $(n-1)$-leg together with the $(n-1)$-leg would correspond to a positive 1 -fold. This can only be a $C P^{1}$, so the entire circlecomplement can be replaced by a single circle with a double leg connecting it to the dot-complement instead of the $(n-1)$-leg. But then the configuration was not minimal.

Proposition 4. The dot-complement of a decomposing 0-leg in a minimal diagram is:

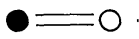

Proof. We reverse the roles of the two complements in the previous argument.

\section{The Polynomial Deformation Method}

For the purpose of PDM, we consider a compact complex manifold $\mathscr{W}$. Let $\mathscr{E}$ be a vector bundle over $\mathscr{W}$ and $v$ a section of $\mathscr{E}$ such that $\mathscr{M}:=v^{-1}(0)$ is a non-singular submanifold of $\mathscr{W}$. In terms of configurations, $\mathscr{W}=\prod_{r=1}^{m} C P_{r}^{n_{r}}$ and $v$ is identified
with a set of constraint polynomials:

$$
\left\{I^{a} \equiv \sum_{r=1}^{m} \alpha_{a}^{\mu r}\left(z_{\mu r}\right)^{q_{a}^{r}}\right\}_{a=1, \ldots, h},
$$

where $\alpha$ 's $\in C, z_{\mu r}$ is the $\mu^{\text {th }}$ homogeneous coordinate of $C P_{r}^{n_{r}}, h=\operatorname{dim} \mathscr{W}-3$ and the configuration matrix $\mathbf{q}$, represents the deformation class of $\mathscr{M}_{\mathrm{CY}}$ defined by $I^{a}=0$. $\mathscr{E}$ is the direct sum of the line bundles determined by the degrees of homogeneity of the constraints.

An appropriate quotient of the space of all sections of $\mathscr{E}$ with non-singular zero-set defines a space of manifolds $\mathscr{M}$ embedded in $\mathscr{W}$ on which the connected component of the group of automorphisms of $\mathscr{W}$ acts. We define $\mathfrak{D}(\mathscr{M}, \mathscr{W})$ to be the orbit space of this action.

PDM, as formulated in the physics literature $[2,8]$, relies on the assumption that $\searrow(\mathscr{M}, \mathscr{W})$ is a complete effective space of deformations of $\mathscr{M}$ in the sense of [9].

In the case of Calabi-Yau manifolds there exists the bundle isomorphism:

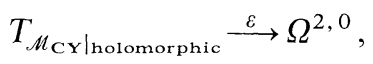


where $\varepsilon$ is the holomorphic 3-form, and the analogous one for the antiholomorphic tangent bundle (in other words, a contravariant vector is isomorphic to the antisymmetric product of two covariant ones.) Consequently, we have that ${ }^{2}$ :

$$
H^{q}\left(\mathscr{M}_{\mathrm{CY}}, T_{\mathscr{M}_{\mathrm{CY}}}\right) \stackrel{\varepsilon}{\approx} H^{q}\left(\mathscr{M}_{\mathrm{CY}}, \Omega^{2,0}\right)=: H^{2, q}\left(\mathscr{M}_{\mathrm{CY}}\right) \text {. }
$$

Thus, the number of deformations, $\eta=\operatorname{dim} \mathfrak{D}\left(\mathscr{M}_{\mathrm{CY}}, \mathscr{W}\right)$, should equal $b_{1,2}=b_{2,1}$ $\equiv H^{2,1}\left(\mathscr{M}_{\mathrm{CY}}\right)$. For a $\mathscr{M}_{\mathrm{CY}}, b_{p, q}=1$ if both $p$ and $q=0(\bmod 3)$, and $b_{p, q}=0$ if exactly one of $p, q=0(\bmod 3)$. Since $b_{1,2}=b_{2,1}$ and $b_{1,1}=b_{2,2}$ by duality, $\chi_{E}=2\left(b_{1,1}\right.$ $\left.-b_{1,2}\right)$, and so if $b_{1,2}$ is calculable, $b_{1,1}$ is given as well.

To obtain a basis for the tangent space to $\mathfrak{D}\left(\mathscr{M}_{\mathrm{CY}}, \mathscr{W}\right)$, one considers variations of the coefficients $\alpha$ in Eq. (2), and divides out the subspace generated by coordinate transformations of $\mathscr{W}$ as well as by "rescalings" of each $I^{a}$ by appropriate combinations of $I^{b}$ 's. To clarify this procedure, we give a few simple, yet non-trivial examples. Subscripts stand for $\chi_{E}$ and superscripts denote $b_{1,1}$. We also show, for purposes of illustration, the diagram corresponding to each configuration.

Let our first example be:

$$
\mathscr{M}_{1} \in\left(\begin{array}{l||ll}
3 & 2 & 2 \\
2
\end{array} \mid \begin{array}{ll}
2 & 2
\end{array}\right)^{2} \cong
$$

$I^{1}$ of $\mathscr{M}_{1}$ is quadratic in the (four) homogeneous coordinates of $C P^{3}$ and linear in those of $C P^{2}$, having thus $\left(\frac{4 \cdot 5}{1 \cdot 2}\right)\left(\frac{3}{1}\right)=30$ coefficients. Since the definition of $\mathscr{M}_{\mathrm{CY}}$ requires $I^{1}=0$, one of them can be rescaled to $1 . I^{2}$ has, analoguously, $\left(\frac{4 \cdot 5}{1 \cdot 2}\right)\left(\frac{3 \cdot 4}{1 \cdot 2}\right)$ $-1=59$ coefficients, after the rescaling. However, the three terms $z_{v 2} \cdot I^{1}$ $\left(z_{v 2} \in C P^{2}\right)$, that vanish whenever $I^{1}$ does, can be added to $I^{2}$ multiplied by appropriate constants to "absorb" three of the 59 coefficients (we refer to this as "embedding" of $I^{1}$ into $I^{2}$. Finally, the 23-dimensional group of coordinate transformations, $P G L(4 ; C) \times P G L(3 ; C)$, is used to rescale 23 of the coefficient to 1 , leaving altogether 62 "effective deformations" [9] of $\mathfrak{D}\left(\mathscr{M}_{1}, \mathscr{W}_{1}\right)$. This agrees with $b_{2,1}=b_{1,1}-\frac{1}{2} \chi_{E}=62$.

In the second example,

$$
\mathscr{M}_{2} \in\left(\begin{array}{l||lll}
3 & 1 & 1 & 2 \\
3 & 1 & 1 & 2
\end{array}\right)^{2} \cong \|_{0}
$$

both $I^{1}$ and $I^{2}$ have $\left(\frac{4}{1}\right)\left(\frac{4}{1}\right)-1=15$ coefficients, but this time they can be "embedded" into each other, reducing the number of coefficients in each of them to 14. $I^{3}$ has $\left(\frac{4 \cdot 5}{1 \cdot 2}\right)\left(\frac{4 \cdot 5}{1 \cdot 2}\right)-1=99$ coefficients, but this time one can "embed" $I^{1}$ into $I^{3}$ in 16 ways (since $z_{\mu 1} z_{v 2} \cdot I^{1} \leadsto I^{3}$ and $\mu, v=0, \ldots, 3$ ) reducing the number to 83 . One can, further, "embed" $I^{2}$ into $I^{3}$ as well, but this time there are only 15 terms, since one linear combination of $z_{\mu 1} z_{\mu 2} \cdot I^{2}$ terms will precisely match the embedding of $I^{1}$ and would therefore be double-counted. Recollecting, we have so far $14+14+68$ $=96$ coefficients. 30 of these can be rescaled to 1 by $P G L(4 ; C) \times P G L(4 ; C)$ coordinate transformations. This yields 66 "effective deformations" of $D\left(\mathscr{M}_{2}, \mathscr{W}_{2}\right)$, matching $b_{1,2}=b_{1,1}-\frac{1}{2} \chi_{E}$.

\footnotetext{
${ }^{2}$ We shall denote and isomorphism by " $\approx$ " and use " $\cong "$ for an equivalence
} 
Consider now the following example:

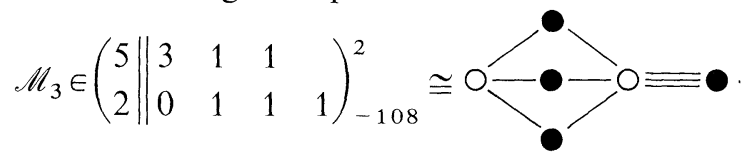

There are $\left(\frac{6 \cdot 7 \cdot 8}{1 \cdot 2 \cdot 3}\right)-1+3 \cdot\left(\frac{6}{1} \cdot \frac{3}{1}-1\right)=106$ coefficients, but actually the latter three can be "embedded" one into another to rescale 6 of them. Using the coordinate transformations of $C P^{5} \times C P^{2}$, one rescales 43 more, yielding $\operatorname{dim} D\left(\mathscr{M}_{3}, \mathscr{W}_{3}\right)=57$, while using straightforward methods $[4,10]$ one obtains the values for $\chi_{E}$ and $b_{1,1}$ as displayed above, and thus $b_{1,2}=56$. As a consequence of this overcounting, PDM would yield a fake element for $H^{2,1}\left(\mathscr{M}_{3}\right)$. Fortunately, it is possible to refine PDM by noting ${ }^{3}$ that the condition for the last three constraint equations to have a non-trivial solution in $C P^{2}$ is that

$$
\operatorname{det}\left\{\partial I^{a} / \partial z_{\mu}\right\}=0, \quad a=2,3,4 ; \quad z_{\mu} \in C P^{2} .
$$

This is, however, a cubic holomorphic and homogeneous equation in the variables of $C P^{5}$ and can therefore be embedded into $I^{1}$, leading to $\operatorname{dim} D\left(\mathscr{M}_{3}, \mathscr{W}_{3}\right)=56$, which is the correct number. It should nevertheless be clear at this point that a more detailed analysis is of PDM is desirable.

Finally, in the fourth example,

$$
\mathscr{M}_{4} \in\left(\begin{array}{l||ll}
4 \\
1
\end{array} \| \begin{array}{ll}
4 & 1 \\
0 & 2
\end{array}\right)_{-168}^{2} \cong(\|)_{O} \|_{-}^{O}
$$

we find $\left(\frac{5 \cdot 6 \cdot 7 \cdot 8}{1 \cdot 2 \cdot 3 \cdot 4}-1\right)+\left(\frac{5}{1} \cdot \frac{2 \cdot 3}{1 \cdot 2}-1\right)=83$ coefficients, 27 of which can be rescaled by the coordinate transformations of $C P^{4} \times C P^{1}$. This yields $\operatorname{dim} \mathfrak{D}\left(\mathscr{M}_{4}, \mathscr{W}_{4}\right)=56$ and so PDM fails to parametrize 30 of the elements of $H^{2,1}\left(\mathscr{M}_{4}\right)$, since $b_{1,2}=86$. One may hope that at least the 56 forms are parametrized irredundantly, but at this point therre is no obvious way of checking that. Actually our explicit analysis of this example, presented in the Appendix B, reveals that even this is not true; not only does PDM give an incomplete parametrization, but it also yields fake elements for $H^{2,1}\left(\mathscr{M}_{4}\right)$.

We wish to emphasize that it is indeed possible that there may exist an altogether different embedding of this $\mathscr{M}_{\mathrm{CY}}$ (constructed here as $\mathscr{M}_{4}$ ), such that PDM yields a correct parametrization of $H^{2,1}\left(\mathscr{M}_{\mathrm{CY}}\right)$ but that is not the issue. Rather, we shall provide an analysis of PDM which will apply to all configurations, and will provide a correct computation in many cases when PDM is invalid.

In the literature $[2,8]$ this construction is completed for some simple configurations, identifying the basis of the tangent space to $D\left(\mathscr{M}_{\mathrm{CY}}, \mathscr{W}\right)$ represented (typically) by monomials in coordinates of $\mathscr{W}$, with a basis of $H^{2,1}\left(\mathscr{M}_{\mathrm{CY}}\right)$. In addition, using the explicit form of $\left\{I^{a}\right\}$, discrete symmetries of $\left\{I^{a}\right\}=0$ and thereby of $\mathscr{M}_{\mathrm{CY}}$ were found together with their action on the coordinates of $\mathscr{W}$. This, in turn, specifies the symmetry property of each particular monomial in $\mathfrak{D}\left(\mathscr{M}_{\mathrm{CY}}, \mathscr{W}\right)$ and thereby - provided $\mathrm{D}\left(\mathscr{M}_{\mathrm{CY}}, \mathscr{W}\right)$ is complete and effective for the case considered - the symmetry property of each corresponding $(2,1)$-form. Thereupon, the

${ }^{3}$ We are indebted to M. Cvetič and V. Kaplunovski for pointing this out to us 
Wigner-Eckart theorem can be used to determine relative values of the Yukawa couplings, and in particular, if they vanish.

In order to see both the obstructions to validity of the computation we have just outlined [i.e. the possible incompleteness or ineffectiveness of $\mathcal{D}(\mathscr{M}, \mathscr{W})]$ and its essential independence of deformation theory, let us reformulate it:

We recall that the derivative of the section $v$ is well defined [10] at each point $p \in \mathscr{M}$ since $v(p)=0$, and gives a linear map, $D_{p} v$ from $T_{p}(\mathscr{W})$ to $\mathscr{E}_{p}$, the fibre of $\mathscr{E}$ at $p$. Since $v^{-1}(0)$ is non-singular at $p$, then $\operatorname{Im} D_{p} v=\mathscr{E}$ and $D_{p} v=T_{p}(\mathscr{M})$. This is summarized by the short exact sequence:

where $j$ corresponds to $D_{p} v$.

$$
\left.\left.0 \longrightarrow T_{\mathscr{M}} \stackrel{i}{\longrightarrow} T_{\mathscr{W}}\right|_{\mathcal{M}} \stackrel{j}{\longrightarrow} \mathscr{E}\right|_{\mathcal{M}} \longrightarrow 0
$$

This gives rise to the following cohomology diagram:

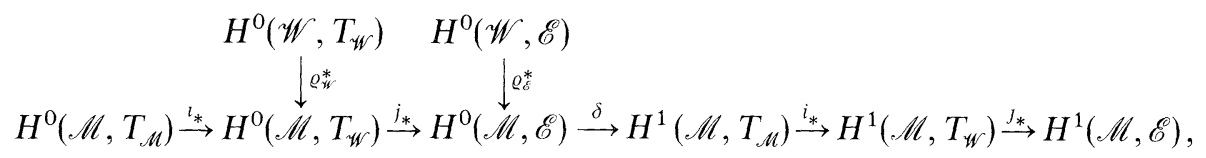

where the bottom row is exact and $\varrho$ is the map restricting $\mathscr{W} \rightarrow \mathscr{M}$.

Clearly, $\delta$ induces a 1-map:

$$
\delta_{1}: H^{0}(\mathscr{M}, \mathscr{E}) /\left(j_{*} H^{0}\left(\mathscr{M}, T_{\mathscr{W}}\right)\right) \rightarrow H^{1}\left(\mathscr{M}, T_{\mathscr{M}}\right),
$$

which is an isomorphism provided $j_{*}: H^{1}\left(\mathscr{M}, T_{\mathscr{W}}\right) \rightarrow H^{1}(\mathscr{M}, \mathscr{E})$ is 1-1.

The diagram also induces a map:

$$
\delta_{2}: \varrho_{\mathscr{E}}\left(H^{0}(\mathscr{W}, \mathscr{E})\right) /\left\{j_{*} \circ \varrho_{\mathscr{W}}\left(H^{0}\left(\mathscr{W}, T_{\mathscr{W}}\right)\right) \cap \varrho_{\mathscr{E}}\left(H^{0}(\mathscr{W}, \mathscr{E})\right)\right\} \rightarrow H^{1}\left(\mathscr{M}, T_{\mathscr{M}}\right)
$$

the coset on the left-hand side being what is computed in PDM. Now we see that what is commonly assumed in the considerations that we sketched in the first part of this section is that $H^{1}\left(\mathscr{M}, T_{\mathscr{M}}\right)$ may be parametrized by the domain of $\delta_{2}$, calculated by PDM, i.e. that $\delta_{2}$ is an isomorphism. This can fail to be true in three ways:

1. $\varrho_{\mathscr{W}}$ is not onto $\Leftrightarrow \delta_{2}$ is not $1-1, \mathfrak{D}(\mathscr{M}, \mathscr{W})$ is ineffective;

2. $\varrho_{\mathscr{E}}$ is not onto $\Rightarrow \delta_{2}$ is not onto, $\mathfrak{D}(\mathscr{M}, \mathscr{W})$ is incomplete;

3. $j_{*}: H_{1}\left(\mathscr{M}, T_{\mathscr{W}}\right) \rightarrow H^{1}(\mathscr{M}, \mathscr{E})$ is not $1-1 \Rightarrow \delta_{2}$ is not onto, $\mathfrak{D}(\mathscr{M}, \mathscr{W})$ is incomplete.

Therefore, even if $\operatorname{dim} \mathfrak{D}(\mathscr{M}, \mathscr{W})=\operatorname{dim} H^{1}\left(\mathscr{M}, T_{\mathscr{M}}\right)$, there could be both overcounting and undercounting. We note that only the interpretation in the last column, which is essentially irrelevant to the computation, depends in any way on the Kodaira-Spencer deformation theory.

\section{The Spectral Sequences}

It is clear that in order to carry the analysis of PDM beyond the point we reached in the previous section, it is necessary to study the vertical maps $\varrho^{*}$ of diagram (7).

We begin by pointing out that by cohomology groups with coefficients in a holomorphic bundle over a complex manifold we mean the cohomology with values in the sheaf of germs of holomorphic sections of the given bundle. By the 
Dolbeault theorem, this is equivalent to the $\bar{\partial}$-cohomology which physicists generally have in mind. In particular, if no coefficient bundle is specified, $H^{q}(\mathscr{M})$ means the cohomology of $\mathscr{M}$ with coefficients in the sheaf of germs of holomorphic functions on $\mathscr{M}$ which is equivalent, by the Dolbeault theorem, to $H^{0, q}(\mathscr{M})$.

We consider the restriction $\varrho^{*}: H^{*}(\mathscr{W}) \rightarrow H^{*}(\mathscr{M})$. Recalling that $v$ is the section of $\mathscr{E}$ which defines $\mathscr{M}$ as its 0 -set (as described in the beginning of Sect. 3), we write the sequence of sheaves:

$$
\vartheta_{\mathscr{W}}\left(\mathscr{E}^{*}\right) \stackrel{v}{\longrightarrow} \vartheta_{\mathscr{W}} \stackrel{\varrho}{\longrightarrow} \vartheta_{\mathcal{M}} \longrightarrow 0 .
$$

Here $\vartheta_{\mathscr{W}}\left(\mathscr{E}^{*}\right)$ denotes the sheaf of germs of holomorphic sections of $\mathscr{E} *$ over $\mathscr{W}$ and $\vartheta_{\mathscr{W}}$ and $\vartheta_{\mathscr{M}}$ denote respectively the sheaves of germs of holomorphic functions on $\mathscr{W}$ and $\mathscr{M} . \vartheta_{\mathcal{M}}$ may be interpreted as a sheaf over $\mathscr{W}$ by assigning to each open set $U \subset \mathscr{W}$, the holomorphic functions on $U \cap \mathscr{M}$. The cohomology of $\mathscr{W}$ with coefficients in this sheaf is the same as the sheaf cohomology of $\mathscr{M}$ in the usual sense.

With this interpretation, (10) becomes a sequence of sheaves over $\mathscr{W}$ which is exact at $\vartheta_{\mathcal{M}}$ and at $\vartheta_{\mathscr{W}}$. We shall hereafter use the same symbol for a vector bundle and the corresponding sheaf [e.g. $\mathscr{E}$ will stand for $\vartheta_{W}(\mathscr{E})$ as well].

In order to motivate what will follow, let us temporarily restrict ourselves to the case in which $\mathscr{E}$ is one dimensional (i.e. there is only one constraint). In this case, (10) can be extended to the short exact sequence:

$$
0 \longrightarrow \mathscr{E}^{*} \stackrel{v}{\longrightarrow} \vartheta_{\mathscr{W}} \stackrel{\varrho}{\longrightarrow} \vartheta_{\mathcal{M}} \longrightarrow 0 .
$$

We may also tensor with $\mathscr{E}$ and $T_{\mathscr{W}}$ to obtain the short exact sequences:

$$
\left.0 \longrightarrow \vartheta_{\mathscr{W}} \stackrel{v}{\longrightarrow} \mathscr{E} \stackrel{\varrho_{\mathscr{E}}}{\longrightarrow} \mathscr{E}\right|_{\mathcal{M}} \longrightarrow 0
$$

and

$$
\left.0 \longrightarrow \mathscr{E} * \otimes T_{\mathscr{W}} \stackrel{v}{\longrightarrow} T_{\mathscr{W}} \stackrel{Q_{W}}{\longrightarrow} T_{\mathscr{W}}\right|_{\mathcal{M}} \longrightarrow 0,
$$

each of which may also be interpreted as a sequence of sheaves over $\mathscr{W}$ in the manner explained above.

Since we are interested in Calabi-Yau manifolds, let us restrict further to the case when $\mathscr{W}$ is a product of projective spaces of total dimension 4 , and $\mathscr{E} *$ is the canonical bundle, i.e. the bundle of $(4,0)$ forms.

Then the sequence (11) yields the isomorphism:

$$
\begin{gathered}
H^{0}(\mathscr{M}) \approx H^{0}(\mathscr{W}) \approx \mathbf{C}, \\
H^{3}(\mathscr{M}) \approx H^{4}(\mathscr{W}, \mathscr{E} *) \approx H^{4,4}(\mathscr{W}) \approx \mathbf{C},
\end{gathered}
$$

and $H^{q}(\mathscr{M})$ vanishes otherwise. This is easily seen from the long exact sequence of cohomology groups, where one knows that $H^{q}(\mathscr{W}, \mathscr{E} *)$ vanishes except for $q=4$, and that $H^{q}(\mathscr{W})$ vanishes except for $q=0$.

By similar arguments, sequence (12) tells us that $\varrho_{\mathscr{E}}: H^{0}(\mathscr{W}, \mathscr{E}) \rightarrow H^{0}(\mathscr{M}, \mathscr{E})$ is onto since $H^{1}(\mathscr{W}) \approx H^{0,1}(\mathscr{W})=0$. It also provides an isomorphism: $H^{q}(\mathscr{W}, \mathscr{E})$ $\approx H^{q}(\mathscr{M}, \mathscr{E}), \mathrm{q}>0$.

The analysis of sequence (13) requires some information about the cohomology of projective spaces [11], which we list here as: 
Bott Vanishing Theorem 1 [BVT1]. $H^{q}\left(C P^{n}, \lambda^{k}\right)$ vanishes except for the following cases:
1. $q=0$ and $k \geqq 0$,
2. $q=n$ and $k<-n$.

Bott Vanishing Theorem 2 [BVT2]. $H^{q}\left(C P^{n}, \xi \otimes \lambda^{k}\right)$, with $k \geqq-(n+1)$, vanishes except for the following cases:

1. $q=0$ and $k \geqq-1$,

2. $q=n-1$ and $k=-(n+1)$.

Here $\lambda$ is the Hopf bundle over $C P^{n}$ (i.e. the bundle the sections of which correspond to homogeneous linear polynomials), and we abbreviate $T\left(C P^{n}\right)$ to $\xi$.

Now by writing $\mathscr{W}=\prod_{s} C P_{s}^{n_{s}}, N:=\sum n_{s}=4$, and observing that $T_{\mathscr{W}}=\bigoplus_{s} \xi_{s}$, we see that the cohomology of the middle term of sequence (13) vanishes except for $H^{0}\left(\mathscr{W}, T_{\mathscr{W}}\right)=\bigoplus_{s} H^{0}\left(C P_{s}^{n_{s}}, \xi_{s}\right)$. Since $\mathscr{E}$ is in this case $\underset{s}{\otimes} \lambda_{s}^{\left(n_{s}+1\right)}$, we have:

$$
\begin{aligned}
H^{j}\left(\mathscr{W}, \mathscr{E} * \otimes T_{\mathscr{W}}\right)= & \bigoplus_{r=1}^{m} \bigoplus_{\Sigma \gamma_{t}=j}\left[H^{\gamma_{r}}\left(C P_{r}^{n_{r}}, \xi_{r} \otimes\left(\lambda_{r}\right)^{-\left(n_{r}+1\right)}\right)\right. \\
& \left.\otimes \substack{\begin{subarray}{c}{s=1 \\
s \neq r} }} \\
{m} \end{subarray}^{m} H^{\gamma_{s}}\left(C P_{s}^{n_{s}},\left(\lambda_{s}\right)^{-\left(n_{s}+1\right)}\right)\right] .
\end{aligned}
$$

Noting that:

$$
H^{n_{s}}\left(C P_{s}^{n_{s}}, \lambda_{s}^{-\left(n_{s}+1\right)}\right)=H^{n_{s}, n_{s}}\left(C P_{s}^{n_{s}}\right) \approx \mathbf{C},
$$

we see that $H^{3}\left(\mathscr{W}, T_{\mathscr{W}} \otimes \mathscr{E}^{*}\right)$ has $\operatorname{rank} m$, and $H^{q}\left(\mathscr{W}, T_{\mathscr{W}} \otimes \mathscr{E}^{*}\right.$ vanishes otherwise.

In particular, $H^{1}\left(\mathscr{W}, T_{\mathscr{W}} \otimes \mathscr{E}^{*}\right)=0$, so that $\varrho_{\mathscr{W}}^{*}: H^{0}\left(\mathscr{W}, T_{\mathscr{W}}\right) \rightarrow H^{0}\left(\mathscr{M}, T_{\mathscr{W}}\right)$ is onto. Moreover, $H^{1}\left(\mathscr{M}, T_{\mathscr{W}}\right)=0$ and $H^{2}\left(\mathscr{W}, T_{\mathscr{W}}\right)$ has rank $m$.

This is sufficient to establish the validity of PDM for the case of a single constraint. We note also that $H^{2}\left(\mathscr{W}, T_{\mathscr{W}}\right) \approx H^{2,2}(\mathscr{M})$, so we have established that $b_{1,1}(\mathscr{M})=b_{2}(\mathscr{M})=b_{2,2}(\mathscr{M})=m$, which could also have been derived from the Lefschetz Hyperplane Theorem in this case [10].

Returning to the general case of an indecomposable configuration representing a Calabi-Yau 3-fold ${ }^{4}$, we wish to extend the exact sequence (10) to the left so that it remains exact. Such an extension is called a resolution of the restriction $\varrho: \vartheta_{\omega} \rightarrow \vartheta_{\mathscr{M}}$, and is accomplished by writing:

$$
0 \longrightarrow \wedge^{h} \mathscr{E} * \stackrel{v}{\longrightarrow} \ldots \stackrel{v}{\longrightarrow} \wedge^{2} \mathscr{E} * \stackrel{{ }^{\prime}}{\longrightarrow} \mathscr{E} * \stackrel{\nu}{\longrightarrow} \vartheta_{\mathscr{W}} \stackrel{\varrho}{\longrightarrow} \vartheta_{\mathcal{M}} \longrightarrow 0
$$

where each map but the last is induced by the section $v$ of $\mathscr{E}$ acting on the last factor of $\wedge^{k} \mathscr{E} *$, and $h$ is $\operatorname{dim} \mathscr{E}$, the number of constraints. The sequence is clearly exact as a sequence of bundles except along $\mathscr{M}$. It gives an exact sequence of sheaves over $\mathscr{W}$ since no non-empty open subset of $\mathscr{W}$ is contained in $\mathscr{M}$.

\footnotetext{
${ }^{4}$ With minor modifications, our present analysis actually applies in a much wider context. For the sake of simplicity, we confine ourselves to the case of most immediate physical interest
} 
We analyze this situation by extracting the short exact sequences:

$$
\begin{gathered}
0 \rightarrow v\left(\wedge^{k+1} \mathscr{E}^{*}\right) \rightarrow \wedge^{k} \mathscr{O}^{*} \rightarrow v\left(\wedge^{k} \mathscr{E} *\right) \rightarrow 0, \\
0 \rightarrow v\left(\mathscr{E}^{*}\right) \rightarrow \vartheta_{\mathscr{W}} \stackrel{\varrho^{*}}{\rightarrow} \vartheta_{\mathcal{M}} \rightarrow 0 .
\end{gathered}
$$

These sequences induce boundary operators:

$$
\partial_{0}: H^{q}(\mathscr{M}) \rightarrow H^{q+1}\left(v\left(\mathscr{E}^{*}\right)\right) \quad \text { and } \partial_{k}: H^{q}\left(\wedge^{k} \mathscr{E} *\right) \rightarrow H^{q+1}\left(v\left(\wedge^{k+1} \mathscr{E} *\right)\right) .
$$

Observing now that $\operatorname{ker} \partial_{0}=\operatorname{Im} \varrho^{*}$, we write:

$$
\mathscr{F}_{k} H^{q}(\mathscr{M}):=\operatorname{ker}\left(\partial_{k} \circ \ldots \circ \partial_{0}\right): H^{q}(\mathscr{M}) \rightarrow H^{q+k+1}\left(\wedge^{k+1} \mathscr{E} *\right) .
$$

Thus $\mathscr{F}_{k} H^{q}(\mathscr{M}) \subset \mathscr{F}_{k+1} H^{q}(\mathscr{M}), \mathscr{F}_{0} H^{q}(\mathscr{M})=\operatorname{Im} \varrho^{*}$ and $\mathscr{F}_{h} H^{q}(\mathscr{M})=H^{q}(\mathscr{M})$. This establishes an increasing filtration:

$$
\varrho\left[H^{q}(\mathscr{W})\right]=: \mathscr{F}_{0} H^{q}(\mathscr{M}) \subseteq \mathscr{F}_{1} H^{q}(\mathscr{M}) \subseteq \ldots \subseteq \mathscr{F}_{h} H^{q}(\mathscr{M}):=H^{q}(\mathscr{M}) .
$$

The foregoing analysis clearly applies equally well if we tensor the entire resolution (15) with any fixed holomorphic vector bundle $\mathscr{V}$ over $\mathscr{W}$.

We now describe a computational tool which is available for this kind of situation:

Definition [12]. A spectral sequence is a sequence $\left\{E_{i}, d_{i}\right\}(i>0)$ of bi-graded groups $E_{i}=\bigoplus_{j, k} E_{i}^{j, k}$ together with differentials

$$
d_{i}: E_{i}^{j, k} \rightarrow E_{i}^{j+J(i), k+K(i)}, \quad d_{i} \circ d_{i}=0,
$$

such that $E_{i+1}=H^{*}\left(E_{i}, d_{i}\right)$, i.e. $E_{i+1}^{j, k}$ consists of the elements of $E_{i}^{j, k}$ that are $d_{i^{-}}$ closed, modulo those that are $d_{i}$-exact.

While $E_{i+1}$ is determined by $E_{i}$ and $d_{i}, d_{i+1}$ is not. In practice, as we shall see in the sequel, spectral sequences are most useful when most of the differentials have null domain or range, and the remaining ones can be deduced indirectly.

A spectral sequence is said to converge if for each $j, k$ there exists a $\hat{\imath}(j, k)$ so that

$$
E_{i}^{j, k}=E_{i+1}^{j, k}=: E_{\infty}^{j, k}, \quad i>\hat{\imath}(j, k) .
$$

Just as in the case of (11), the exactness of (15) is preserved under the tensor product with any vector bundle $\mathscr{V}$ over $\mathscr{W}$, and induces a spectral sequence $\left\{E_{i}\left(\mathscr{V}, d_{i}\right)\right\}$ with the following properties (see Godement in [12]):

1. $E_{1}^{j, k}(\mathscr{V})=H^{j}\left(\mathscr{W}, \mathscr{V} \otimes \wedge^{k} \mathscr{E} *\right), d_{1}:=v_{*} ;$

2. $d_{i}: E_{i}^{j, k}(\mathscr{V}) \rightarrow E_{i}^{j-i+1, k-i}(\mathscr{V}) ; \Rightarrow d_{i} \equiv 0$ for $i>h$, and so $E_{h+1}(\mathscr{V})=E_{\infty}(\mathscr{V})$;

3. $H^{j}(\mathscr{M}, \mathscr{V})$ has an increasing filtration such that:

- $\mathscr{F}_{0} H^{j}(\mathscr{M}, \mathscr{V})=\varrho\left[H^{i}(\mathscr{W}, \mathscr{V})\right]$,

- $\mathscr{F}_{i} H^{j}(\mathscr{M}, \mathscr{V}) / \mathscr{F}_{i-1} H^{j}(\mathscr{M}, \mathscr{V}) \approx E_{\infty}^{j+i, i}(\mathscr{V})$.

Representing a spectral sequence by a chart is often very useful and the specific one that we consider here is given in Fig. 1 . The action of the differentials $d_{i}$ is indicated by the arrows. This action maps the groups $E_{i}^{j, k}$ with $j-k=q$, to which we refer as "on the $q$-diagonal", into those on the $(q+1)$-diagonal. 


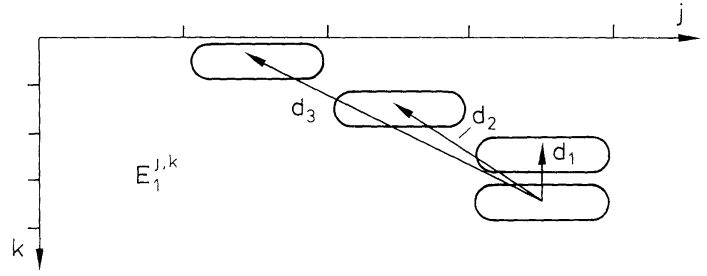

Fig. 1. The chart representing the spectral sequence

It should be noted that the various groups $E_{\infty}^{q+k, k}(\mathscr{V})$ determine only the successive quotients $\mathscr{F}_{i} H^{j}(\mathscr{M}, \mathscr{V}) / \mathscr{F}_{i-1} H^{j}(\mathscr{M}, \mathscr{V})$, not $\mathscr{F}_{i} H^{j}(\mathscr{M}, \mathscr{V})$ themselves. This is enough, however, to obtain:

$$
\operatorname{dim} H^{q}(\mathscr{M}, \mathscr{V})=\sum_{k=0}^{h} \operatorname{rank} E_{\infty}^{q+k, k}(\mathscr{V}),
$$

which, in a sense, corresponds to collecting all the groups $E_{\infty}^{j, k}$ on the $q$-diagonal, even though there is no natural isomorphism of $H^{q}(\mathscr{M}, \mathscr{V})$ with $\bigoplus_{k=0}^{h} E_{\infty}^{q+k, k}(\mathscr{V})$.

In the case when, for some $q, E_{\infty}^{q+k, k}(\mathscr{V})$ vanishes for all $k>0$, we have $E_{\infty}^{q, 0}(\mathscr{V})$ $\approx \mathscr{F}_{0} H^{q}(\mathscr{M}, \mathscr{V}) \approx H^{q}(\mathscr{M}, \mathscr{V})$, and thus $\varrho^{*}\left[H^{q}(\mathscr{W}, \mathscr{V})\right] \approx H^{q}(\mathscr{M}, \mathscr{V})$. Similarly when, for some $q, E_{\infty}^{q+k, k}$ vanishes for all but a single $k=\kappa, H^{q}(\mathscr{M}, \mathscr{V}) \approx E_{\infty}^{q+\kappa, \kappa}(\mathscr{V})$.

Further, since cohomology groups of negative dimension vanish identically, one knows that $E_{\infty}^{j, k}(\mathscr{V})$ vanish for $j<k$. Since $E_{1}^{j, k}(\mathscr{V})$ with $j<k$ may not vanish, this observation may be used to deduce some information about the $d_{i}$ 's.

Finally, if $E_{i}^{j, k}(\mathscr{V})$ vanish on every other diagonal, $E_{i}=E_{\infty}$.

Let us now start with the simplest spectral sequence $\left\{E_{i}, d_{i}\right\}$, the one induced by the exact sequence of sheaves (15). We assume that we are dealing with a configuration so that:

$$
\mathscr{W}=\prod_{s=1}^{m} C P_{s}^{n_{s}} \quad \text { and } \quad \mathscr{E}=\sum_{a=1}^{h} \bigotimes_{s=1}^{m} \lambda_{s}^{q_{a}^{s}} .
$$

To compute $E_{1}^{j, k}$, we write $\mathscr{L}_{a}$ for $\bigotimes_{s=1}^{m} \lambda_{s}^{q_{a}^{s}}$ and note that $\wedge^{k} \mathscr{E}^{*}$ can be decomposed into $\bigoplus_{|A|=k} \bigotimes_{\alpha \in A} \mathscr{L}_{a}^{*}$, where $|A|$ denotes the cardinality of $A$, a subset of indices $a=1, \ldots, h$, so that $\bigoplus_{|A|=k}$ corresponds to the direct sum over all possible choices of $k$ constraints from the set (2). Thus:

$$
E_{1}^{j, k}=\bigoplus_{|A|=k} H^{j}\left(\mathscr{W}, \bigotimes_{s=1}^{m}\left(\lambda_{s}\right)^{-\sum_{a \in A} q_{a}^{s}}\right) .
$$

Finally, using that $\mathscr{W}$ is a product of $C P_{r}^{n_{r}}$, one has:

$$
E_{1}^{j, k}=\bigoplus_{|A|=k} \bigoplus_{\Sigma \gamma_{t}=j}\left[\bigotimes_{s=1}^{m} H^{\gamma_{s}}\left(C P_{s}^{n_{s}},\left(\lambda_{s}\right)^{-\sum_{a \in A} q_{a}^{s}}\right)\right] .
$$


Since vanishing of a (tensor) product is implied by the vanishing of any of its factors, we use BVT1 to establish that very few summands in Eq. (18) do not vanish. In particular, we recover the well-known theorem:

Theorem 1. For a Calabi-Yau 3-fold represented by an indecomposable configuration:

1. $\mathrm{b}_{p, q}=1$ if $p$ and $q \equiv 0(\bmod 3)$;

2. $b_{p, q}=0$ if exactly one of $p, q \equiv 0(\bmod 3)$.

Proof. In order for any subset $A$ to make a non-zero contribution to the summation (18) we must have, for each $s$ :

$$
\text { either } \sum_{a \in A} q_{a}^{s}=0 \text { or } \sum_{a \in A} q_{a}^{s}=n_{s}+1 \text {. }
$$

If $A$ is other than empty or the full index set, this induces a block decomposition of the matrix $\left\{q_{a}^{s}\right\}$. When $A$ is empty, we get a contribution of rank 1 to $E_{1}^{0,0}$. When $A$ is the full index set, we get a contribution of rank 1 to $E_{1}^{N, h}$. Since $N:=\sum n_{s}=h+3$ (we deal with Calabi-Yau manifolds of dimension 3 ), there are no non-trivial differentials in the spectral sequence and $E_{1}=E_{\infty}$. Since $E_{i}^{j, k}$ converges to $H^{j-k}\left(\mathscr{M}, \vartheta_{\mathscr{M}}\right)=H^{0, j-k}(\mathscr{M})$ in this case, it follows that $b_{0,0}=b_{0,3}=1, b_{0,1}=b_{0,2}=0$. The full statements of Theorem 1 follow by complex conjugation or Serre duality.

In the next two sections we shall employ the fact that the exactness of the sequence (15) and therefore the existence of a corresponding spectral sequence is preserved under the tensor product with any vector bundle over $\mathscr{W}$. In particular, we multiply the sequence (15) by $T_{\mathscr{W}}$ or $\mathscr{E}$, and analyze the corresponding spectral sequences, hoping to determine $H^{2,1}\left(\mathscr{M}_{\mathrm{CY}}\right)$ and $H^{2,2}\left(\mathscr{M}_{\mathrm{CY}}\right)$, and thus all $b_{p, q}$. (Note that when this program can be carried out, $\chi_{E}$ is determined independently of the straightforward computation [4] and can thus be checked.)

\section{The Tangent Bundle Spectral Sequence}

We now consider the tensor product of the sequence (15) and $T_{\mathscr{W}}$. The spectral sequence $\left\{E_{i}\left(T_{\mathscr{W}}\right), d_{i}\right\}$ now has typical elements:

$$
E_{1}^{j, k}\left(T_{\mathscr{W}}\right)=H^{j}\left(\mathscr{W}, \wedge^{k} \mathscr{E} * \otimes T_{\mathscr{W}}\right), \ldots
$$

which converge to $H^{j-k}\left(\mathscr{M}, T_{\mathscr{W}}\right)$ appearing in the bottom line of the diagram (7).

We again decompose $T_{\mathscr{W}}$ with respect to the $C P_{s}^{n_{s}}$ 's into $\xi_{s}$ 's and obtain an expansion generalizing that in Eq. (14):

$$
\begin{aligned}
E_{1}^{j, k}\left(T_{\mathscr{W}}\right)= & \bigoplus_{|A|=k} \bigoplus_{r=1}^{m} \bigoplus_{\Sigma \gamma_{t}=J}\left[H^{\gamma_{r}}\left(C P_{r}^{n_{r}}, \xi_{r} \otimes\left(\lambda_{r}\right)^{-\sum_{a \in A} q_{a}^{r}}\right)\right. \\
& \left.\otimes \bigoplus_{\substack{s=1 \\
s \neq r}}^{m} H^{\gamma_{s}}\left(C P_{s}^{n_{s}},\left(\lambda_{s}\right)^{-\sum_{a \in A} q_{a}^{s}}\right)\right] .
\end{aligned}
$$


In order for a pair $A, r$ to make non-zero contribution to the summation (19), we must have:

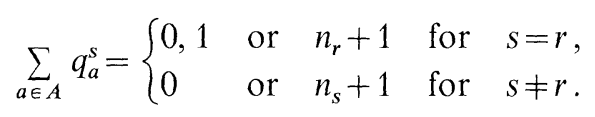

If we have $\sum_{a \in A} q_{a}^{r} \neq 1$ then $A$ is either empty, or the entire index set, just as in the proof of Theorem 1.

When $A$ is empty, we get a contribution of $\sum_{s=1}^{m} H^{0}\left(C P_{s}^{n_{s}}, \xi_{s}\right)$ to $E_{1}^{0,0}\left(T_{\mathscr{W}}\right)$ which corresponds to the usual subtraction of global reparametrizations of $\mathscr{W}$ in PDM.

When $A$ is the entire index set, we get a contribution of rank $k$ (one for each $\left.C P^{n}\right)$ to $E_{1}^{N-1, h}$. This term is on the 2-diagonal and if it survives to $E_{\infty}\left(T_{\mathscr{W}}\right)$, it contributes to $H^{2}\left(\mathscr{M}, T_{\mathscr{W}}\right)$.

The case $\sum_{a \in A} q_{a}^{r}=1$ can occur only if the configuration is $1 \mathrm{LD}$. Each decomposing leg contributes to $E^{\pi+k_{d}, k_{d}}\left(T_{\mathscr{W}}\right)$ with rank [13]:

$$
\operatorname{rank}\left\{H^{0}\left(C P_{r}^{n_{r}}, \xi_{r} \otimes \lambda_{r}^{-1}\right)\right\}=n_{r}+1 \text {. }
$$

Here $\pi=l_{d}-m_{d}-k_{d}$ is the index of the decomposing leg, $C P_{r}^{n_{r}}$ corresponds to the hollow circle touching the leg, $k_{d}=|A|$, and $A$ is the set of constraints corresponding to the dot-complement of the leg.

If there is no decomposing 1-leg, the entire $E_{1}\left(T_{\mathscr{W}}\right)$ term is concentrated on the 0 - and 2-diagonal and thus $E_{1}\left(T_{\mathscr{W}}\right)=E_{\infty}\left(T_{\mathscr{W}}\right)$. In particular, if there exists a decomposing 0-leg but no decomposing 1-leg, $\varrho^{*}: H^{0}\left(\mathscr{W}, T_{\mathscr{W}}\right) \rightarrow H^{0}\left(\mathscr{M}, T_{\mathscr{W}}\right)$ is not onto and $\mathfrak{D}(\mathscr{M}, \mathscr{W})$ is ineffective.

To summarize, we have proved:

Theorem 2. 1. If a configuration representing a Calabi-Yau 3-fold is not 1LD, the only non-vanishing terms of the $T_{\mathscr{W}}$-spectral sequence are $E_{1}^{0,0}\left(T_{\mathscr{W}}\right)$ and $E_{1}^{N-1, h}\left(T_{\mathscr{W}}\right)$, where $N=\operatorname{dim} \mathscr{W}$ and $h$ is the number of constraints.

2. $E_{1}=E_{\infty}$ if there is no decomposing 1-leg.

3. $\mathfrak{D}(\mathscr{M}, \mathscr{W})$ is ineffective for a $1 \mathrm{LD}$ configuration with at least one decomposing 0 -leg and no decomposing 1-leg.

Remark. Incompleteness can, in fact, "mask" ineffectiveness so as to yield $\operatorname{dim} \mathfrak{D}\left(\mathscr{M}_{\mathrm{CY}}, \mathscr{W}\right) \leqq \operatorname{dim} H^{2,1}\left(\mathscr{M}_{\mathrm{CY}}\right)$. As a matter of fact, we have found no counterexample ${ }^{5}$ to this relation. The failure of PDM to provide an irredundant basis of $H^{2,1}\left(\mathscr{M}_{\mathrm{CY}}\right)$ for configurations of Theorem 2 however remains; some of the exterior forms constructed by PDM represent the 0-element of $H^{2,1}(\mathscr{W})$. Examples of Statement 3 of Theorem 2 are given in the Appendix B.

Corollary 1. If a configuration representing a Calabi-Yau 3-fold is not 1LD and PDM yields the correct value for $b_{1,2}$, then PDM yields a valid parametrization of $H^{2,1}(\mathscr{M})$.

\footnotetext{
${ }^{5}$ We thank P. Candelas et al. for providing us with the almost 8000 configurations and corresponding Euler characters generated by computer to exhaust the family of minimal configurations
} 
Among the examples we found in the literature $[2,8]$ not one has a decomposing $0-$ leg, and so the map $\varrho_{\mathscr{W}}$ in (7) indeed is 1-1. Thus PDM yields no fake elements for $H^{1}\left(\mathscr{M}_{\mathrm{CY}}, T_{\mathscr{M}}\right)$ and since $\operatorname{dim} \mathfrak{D}(\mathscr{M}, \mathscr{W})=b_{1,2}$ (as computed by other, straightforward methods), $D(\mathscr{M}, \mathscr{W})$ is both effective and complete in these cases.

For the case of a minimal configuration we can carry the analysis further.

Theorem 3. For a minimal configuration, $E_{1}\left(T_{\mathscr{W}}\right)=E_{\infty}\left(T_{\mathscr{W}}\right)$.

Proof. By Proposition 4, the contribution of a decomposing 0-leg to the tangent bundle spectral sequence for a minimal configuration is to $E_{1}^{1,1}\left(T_{\mathscr{W}}\right)$. Then, by the remarks preceding Theorem 2 and Propositions 2 and 3, the only possible nonvanishing components of $E_{1}\left(T_{\mathscr{W}}\right)$ are $E_{1}^{0,0}\left(T_{\mathscr{W}}\right), E_{1}^{1,1}\left(T_{\mathscr{W}}\right), E_{1}^{2+h, h}\left(T_{\mathscr{W}}\right)$ and $E_{1}^{1+k, k}\left(T_{\mathscr{W}}\right), 0<k<h$, where $h$ is the number of constraints. There are no possible differentials connecting any of these.

\section{The Normal Bundle Spectral Sequence}

Now we analyze the $\left\{E_{i}(\mathscr{E}), d_{i}\right\}$ spectral sequence that is induced by the tensor product of the exact sequence $(15)$ with $\mathscr{E}$ and converges to $H^{*}(\mathscr{M}, \mathscr{E})$. In particular,

$$
E_{1}^{0,0}(\mathscr{E})=H^{0}(\mathscr{W}, \mathscr{E})=\bigoplus_{b=1}^{h}\left[\bigoplus_{s=1}^{m} H^{0}\left(C P_{s}^{n_{s}}, \lambda_{s}^{q_{b}^{s}}\right)\right]
$$

which corresponds to the polynomial deformations in PDM. Similarly:

$$
\begin{aligned}
E_{1}^{0,1}(\mathscr{E}) & =H^{0}(\mathscr{W}, \mathscr{E} * \otimes \mathscr{E})=H^{0}(\mathscr{W}, \operatorname{Hom}(\mathscr{E}, \mathscr{E})) \\
& =\left\{\bigoplus_{b=1}^{h}\left[\bigotimes_{s=1}^{m} H^{0}\left(C P_{s}^{n_{s}}\right)\right]\right\} \otimes\left\{\bigoplus_{\substack{h \\
b=1}}^{\substack{a=1 \\
a \neq b}}\left[\bigotimes_{s=1}^{m} H^{0}\left(C P_{s}^{n_{s}}, \lambda_{s}^{\left(q_{b}^{s}-q_{a}^{s}\right)}\right)\right]\right\} .
\end{aligned}
$$

The first term has rank $h$ and corresponds to overall rescaling subtractions of PDM, while the second one is accounted for in PDM by "embedding" the constraints into each other as described in Sect. 3.

Other terms in $E_{1}(\mathscr{E})$ may be determined by writing:

$$
\wedge^{k} \mathscr{E} * \otimes \mathscr{E}=\bigoplus_{b=1}^{h} \bigoplus_{|A|=k}\left(\bigotimes_{a \in A} \mathscr{L}_{a}^{*}\right) \otimes \mathscr{L}_{b}
$$

Note that if $b \in A$, we get a summand of $\wedge^{k-1} \mathscr{E} *$, and it follows from BVT1 that $H^{*}\left(\mathscr{W}, \wedge^{k-1} \mathscr{E}^{*}\right)$ vanishes for $0<k-1<h$. The case $k-1=h$ does not arise, and the case $k-1=0$ contributes to $E_{1}^{0,1}(\mathscr{E})$ which we have already dealt with. The case $|A|=0$ contributes only to $E_{1}^{0,0}(\mathscr{E})$ which we have also discussed.

Thus we may confine our attention to the case $b \notin A,|A| \geqq 1$, i.e. to the corresponding contribution of:

$$
\bigoplus_{b=1}^{h} \bigoplus_{\substack { \Sigma \gamma_{t}=j \\
\begin{subarray}{c}{|A|=k \\
A \neq b{ \Sigma \gamma _ { t } = j \\
\begin{subarray} { c } { | A | = k \\
A \neq b } }\end{subarray}}^{\oplus}\left[\bigoplus_{s=1}^{m} H^{\gamma_{s}}\left(C P_{s}^{n_{s}},\left(\lambda_{s}\right)^{q_{b}^{s}-\sum_{a \subset A} q_{a}^{s}}\right)\right], \quad 1<k<h
$$

to $E_{1}^{j, k}(\mathscr{E})$ 
For a pair $A, b$ to contribute to a non-vanishing term to $E_{1}(\mathscr{E})$, we must have, by BVT1,

$$
\text { either } q_{b}^{s}-\sum_{a \in A} q_{a}^{s} \geqq 0 \quad \text { or } \quad q_{b}^{s}-\sum_{a \in A} q_{a}^{s}=-\left(n_{s}+1\right)
$$

for each $s$. If $q_{b}^{s} \neq 0$, only the $1^{\text {st }}$ option is possible since $\sum_{a \in A} q_{a}^{s} \leqq \sum_{a=1}^{h} q_{a}^{s}=n_{s}+1$. On the other hand, if $q_{b}^{s}=0$ we clearly must have $\sum_{a \in A} q_{a}^{s}=0$ or $n_{s}+1$.

It follows that the constraint matrix $\left\{q_{a}^{s}\right\}$ can then be re-written in the form:

$$
\left(\begin{array}{llll}
0 & \mathbf{0} & \mathbf{P} & \mathbf{0} \\
0 & \mathbf{0} & \mathbf{0} & \mathbf{Q} \\
B & \mathbf{X} & \mathbf{Y} & \mathbf{Z}
\end{array}\right)
$$

where the column $B$ corresponds to the $b^{\text {th }}$ constraint and $\mathbf{P}$ and $\mathbf{X}$ are matrices with non 0-columns. The set of columns of $\mathbf{P}$ and $\mathbf{X}$ (either of which may be empty), correspond to the constraints of $A$, so that, row-by-row, the entry in $B$ is not smaller than the sum of entries in $\mathbf{X}$ and $\mathbf{Y}$.

Such an array contributes to $E_{1}^{N(\mathbf{P}),|A|}(\mathscr{E})$, where $N(\mathbf{P})$ is the total dimension of the $C P^{n}$ 's corresponding to the rows in the matrix $\mathbf{P}$. Rewriting $\left\{\mathbf{q}_{a}^{s}\right\}$ in all possible ways in the above form completes the listing of non-vanishing contributions to $E_{1}(\mathscr{E})$. Rather than attempting to formulate this algorithmically, we apply it to several examples in the Appendices.

However, we can prove:

Theorem 4. For a minimal diagram representing an $n$-fold, $E_{1}^{q+k, k}(\mathscr{E})$ vanishes for $q \geqq n-1$.

Proof. In the diagram corresponding to the above array, let us denote by $F$ the part containing the dots corresponding to the constraints in $A$ and the circles and legs corresponding to $C P^{n}$ 's for which $\left\{q_{a}^{r}\right\}$ has non-zero entries only within $\mathbf{P}$. $\bar{F}$ denotes the rest of the diagram. For this array to yield a non-vanishing contribution to $E_{1}^{q+k, k}(\mathscr{E})$ for $q \geqq n-1$, we need $l_{F}-m_{F}-h_{F} \geqq n-1$. But, since $l-m$ $-h=n, l_{\bar{F}}-m_{\bar{F}}-h_{\bar{F}} \leqq 1$. For the whole diagram to be connected, $\bar{F}$ must have at least one leg with a free end (connecting into $F$ ) so $l_{\bar{F}}-m_{\bar{F}}-h_{\bar{F}}$ must be at least 1 . But then $\bar{F}$ corresponds to a positive 1-fold which can only be a $C P^{1}$ and thus can be replaced by a single circle connected to $F$ by a double leg; i.e. the original configuration was not minimal (since $\bar{F}$ contained at least the constraint $B$ ).

Corollary 2. If a minimal diagram representing a Calabi-Yau 3-fold has no decomposing 1-leg, $H^{1}\left(\mathscr{M}, T_{\mathscr{W}}\right)$ vanishes and the sequences:

are exact.

$$
\begin{gathered}
0 \rightarrow H^{0}\left(\mathscr{M}, T_{\mathscr{W}}\right) \rightarrow H^{0}(\mathscr{M}, \mathscr{E}) \rightarrow H^{1}\left(\mathscr{M}, T_{\mathscr{M}}\right) \rightarrow 0, \\
0 \rightarrow H^{1}(\mathscr{M}, \mathscr{E}) \rightarrow H^{2}\left(\mathscr{M}, T_{\mathscr{M}}\right) \rightarrow H^{2}\left(\mathscr{M}, T_{\mathscr{W}}\right) \rightarrow 0,
\end{gathered}
$$

The first of these short exact sequences yields $H^{1}\left(\mathscr{M}, T_{\mathscr{M}}\right)$ $=\left\{H^{0}(\mathscr{M}, \mathscr{E}) / H^{0}\left(\mathscr{M}, T_{\mathscr{W}}\right)\right\}$ and allows to compute $b_{1,2}=\operatorname{rank} H^{0}(\mathscr{M}, \mathscr{E})$ - rank $H^{0}\left(\mathscr{M}, T_{\mathscr{W}}\right)$ while the second leads to $b_{1,1}=b_{2,2}=\operatorname{rank} H^{1}(\mathscr{M}, \mathscr{E})$ $+H^{2}\left(\mathscr{M}, T_{\mathscr{W}}\right)$. For a minimal configuration this recovers the bound $[10] b_{1,1} \geqq m$. 
Corollary 3. If a diagram representing a Calabi-Yau 3-fold has no decomposing 1-leg and if, in addition, $E_{\infty}^{1+k, k}(\mathscr{E})$ vanishes for all $k, H^{1}(\mathscr{M}, \mathscr{E})$ vanishes and $H^{2}\left(\mathscr{M}, T_{\mathscr{M}}\right) \approx H^{2}\left(\mathscr{M}, T_{\mathscr{W}}\right)$, i.e. $b_{1,1}=b_{2,2}=\operatorname{rank} H^{2}\left(\mathscr{M}, T_{\mathscr{W}}\right)$.

Corollary 4. The map $d_{2}$ in Rel. (9) is onto if a diagram representing a Calabi-Yau 3-fold has no decomposing 1-leg and if, in addition, $E_{\infty}^{k, k}(\mathscr{E})$ vanishes for all $k>0$. Then $b_{1,2}=b_{2,1}=\operatorname{dim} \mathfrak{D}(\mathscr{M}, \mathscr{W})-\operatorname{rank} E_{1}^{1,1}\left(T_{\mathscr{W}}\right)$.

Remark. If a configuration defining a 3-fold has a decomposing 1-leg, our method is, in general not guaranteed to compute $b_{1,2}$ or $b_{1,1}$. In such a case, however, note that incident with the decomposing 1-leg there is a decomposing 2-2-dot. It follows that such a configuration can be studied as a hypersurface in the product of two non-negative 2-folds; we hope to pursue such an analysis in a subsequent paper [14].

\section{Conclusion}

To summarize, we have analyzed the method of polynomial deformations (PDM) as a method of studying $H^{1,2}\left(\mathscr{M}_{\mathrm{CY}}\right)$ of Calabi-Yau manifolds embedded as algebraic varieties in products of complex projective spaces.

We find that PDM definitely provides a redundant parametrization for $H^{1,2}\left(\mathscr{M}_{\mathrm{CY}}\right)$ in the case of a $1 \mathrm{LD}$ configuration with at least one decomposing 0-leg and no decomposing 1-legs (see end of Sect. 4 for the description of the diagramatic representation of configurations). We also note that the basis for $H^{1.2}\left(\mathscr{M}_{\mathrm{CY}}\right)$, as provided by PDM, is quite often incomplete, but we have not found a criterion which is applicable by inspection.

As a generalization of PDM, we present a cohomology computation that relies on three spectral sequences, and is applicable to all configurations. If the $E_{\infty}$ terms of the spectral sequences can be computed completely, they determine the ranks of the cohomology groups $H^{q}\left(\mathscr{M}_{\mathrm{CY}}, T_{W}\right)$ and $H^{q}\left(\mathscr{M}_{\mathrm{CY}}, \mathscr{E}\right)$ in the bottom row of the diagram (7). Using now the exactness of this sequence and the results of Sect. 4, the ranks of the groups $H^{q}\left(\mathscr{M}_{\mathrm{CY}}, T_{\mathscr{M}_{\mathrm{CY}}}\right)$ are completely determined if, in addition, the configuration has no decomposing 1-leg.

In the cases when the configuration does contain a decomposing 1-leg, $H^{1}\left(\mathscr{M}_{\mathrm{CY}}, T_{\mathscr{W}}\right)$ and $H^{1}\left(\mathscr{M}_{\mathrm{CY}}, \mathscr{E}\right)$ may not vanish and the bottom row of the diagram (7) generally contains too many non-vanishing terms to yield a definite result. It can however set bounds on $b_{1,1}$ and $b_{1,2}$ and combining these with an independent computation of $\chi_{E}$ or $b_{1,1}$ may give sufficient information to determine all the relevant maps. Examples of this are given in Appendix C.

It is tempting to try to construct an explicit parametrization of $H^{1,2}(\mathscr{M})$ and $H^{1,1}(\mathscr{M})$ using the information obtained by the spectral sequences. However, any way of doing this will, in general, involve choices of isomorphisms realizing Eq. (17) and it is not clear at this writing how such choices should be made.

\section{Appendices}

\section{A. An Example in Full Detail}

We present here several examples of the cohomology computation we discuss in Sects. 4-6. Since the examples $\mathscr{M}_{1}$ and $\mathscr{M}_{2}$ do not carry any non-trivial 
information, let us start with $\mathscr{M}_{3}$ :

$$
\mathscr{M}_{3} \in\left(\begin{array}{l||llll}
5 & 3 & 1 & 1 & 1 \\
2 & 0 & 1 & 1 & 1
\end{array}\right)_{-108}^{2} \cong 0
$$

where the numbers beside the dots correspond to the indices of the corresponding columns in the configuration matrix.

First of all, note that this configuration is not $1 \mathrm{LD}$, and therefore, the parametrization obtained by PDM is valid by Corollary 1 , since it gives the correct value of $b_{1,2}$. We present here the spectral sequence computation to provide an easy-to-follow example before we apply it to less trivial cases. The only nonvanishing groups in $\left\{E_{i}\left(T_{\mathscr{W}}\right), d_{i}\right\}$ are $E_{1}^{0,0}\left(T_{\mathscr{W}}\right)$ and $E_{1}^{6,4}\left(T_{\mathscr{W}}\right)$, and thus $E_{1}\left(T_{\mathscr{W}}\right)$ $=E_{\infty}\left(T_{\mathscr{W}}\right)$. Following Eq. (19), they are:

$$
E_{\infty}^{0,0}\left(T_{\mathscr{W}}\right)=\left[H^{0}\left(C P_{1}^{5}, \xi_{1}\right) \otimes H^{0}\left(C P_{2}^{2}\right)\right] \otimes\left[H^{0}\left(C P_{1}^{5}\right) \otimes H^{0}\left(C P_{2}^{2}, \xi_{2}\right)\right],
$$

which is just the set of holomorphic vector-fields on the $C P^{n}$ 's, i.e. the coordinate transformations; the rank of this group is 43 and it provides the only contribution to $H^{0}\left(\mathscr{M}_{3}, T_{W}\right)$. Also:

$$
\begin{aligned}
E_{\infty}^{6,4}\left(T_{\mathscr{W}}\right)= & {\left[H^{4}\left(C P_{1}^{5}, \xi_{1} \otimes \lambda_{1}^{-6}\right) \otimes H^{2}\left(C P_{2}^{2}, \lambda_{2}^{-3}\right)\right] } \\
& \oplus\left[H^{5}\left(C P_{1}^{5}, \lambda_{1}^{-6}\right) \otimes H^{1}\left(C P_{2}^{2}, \xi_{2} \otimes \lambda_{2}^{-3}\right)\right],
\end{aligned}
$$

which is, by duality, equivalent to:

$$
\left[H^{1}\left(C P_{1}^{5}, \Omega^{1}\left(\lambda_{1}^{0}\right)\right) \otimes H^{0}\left(C P_{2}^{2}, \lambda_{2}^{0}\right)\right] \oplus\left[H^{0}\left(C P_{1}^{5}, \lambda_{1}^{0}\right) \otimes H^{1}\left(C P_{2}^{2}, \Omega^{1}\left(\lambda_{2}^{0}\right)\right)\right] .
$$

All of these cohomology groups are one-dimensional and so rank $E_{1}^{6,4}\left(T_{\mathscr{W}}\right)=2$.

This spectral sequence converges to $H^{*}\left(\mathscr{M}_{3}, T_{\mathscr{W}}\right)$ and guarantees that $H^{q}\left(\mathscr{M}_{3}, T_{\mathscr{W}}\right)$ vanishes for $q=1,3$ and is of dimension 43 and 2 for $q=0$ and 2 respectively.

The group $E_{1}^{0,0}(\mathscr{E})$ is easily seen from Eq. (21) to be:

$$
E_{1}^{0,0}(\mathscr{E})=\bigoplus_{b=1}^{4}\left[H^{0}\left(C P_{1}^{5}, \lambda_{1}^{q_{b}^{1}}\right) \otimes H^{0}\left(C P_{2}^{2}, \lambda_{2}^{q \frac{b}{b}}\right)\right],
$$

and counts the number of coefficients in the constraints. The rank of this group is $\left[\left(\begin{array}{l}8 \\ 3\end{array}\right)\right]+3 \cdot\left[\left(\begin{array}{c}6 \\ 1\end{array}\right) \cdot\left(\begin{array}{l}3 \\ 1\end{array}\right)\right]=110$. Similarly, the only non-vanishing term in Eq. (22) is:

$$
\begin{aligned}
E_{1}^{0,1}(\mathscr{E})= & \bigoplus_{b=1}^{4}\left[H^{0}\left(C P_{1}^{5}, \lambda_{1}^{0}\right) \otimes H^{0}\left(C P_{2}^{2}, \lambda_{2}^{0}\right]\right. \\
& \oplus \bigoplus_{\substack { b=2 \\
\begin{subarray}{c}{a=2 \\
a \neq b{ b = 2 \\
\begin{subarray} { c } { a = 2 \\
a \neq b } }\end{subarray}}^{4}\left[H^{0}\left(C P_{1}^{5}, \lambda_{1}^{1-1}\right) \otimes H^{0}\left(C P_{2}^{2}, \lambda_{2}^{1-1}\right)\right],
\end{aligned}
$$

the rank of which is obviously $4 \cdot[1]+3 \cdot 2 \cdot[1]=10$.

There is one more non-vanishing group among $E_{1}^{j, k}(\mathscr{E})$, found by Eq. (24):

$$
E_{1}^{2.3}(\mathscr{E})=\left[H^{0}\left(C P_{1}^{5}, \lambda_{1}^{3-3}\right) \otimes H^{2}\left(V P^{2}, \lambda_{2}^{-3}\right)\right] .
$$

By duality, the second term is equivalent to $H^{0}\left(C P^{2}, \lambda_{2}^{0}\right)$, which has rank 1 , and so the whole contribution is of rank 1 . 
It is clear that $E_{1}^{0,1}(\mathscr{E})$ maps into $E_{1}^{0,0}(\mathscr{E})$ by the action of $d_{1}$, but note also that $d_{3}$ maps $E_{3}^{2,3}(\mathscr{E})=E_{1}^{2,3}(\mathscr{E})$ into $E_{3}^{0,0}(\mathscr{E})=\mathrm{E}_{1}^{0,0}(\mathscr{E}) / E_{1}^{0,1}(\mathscr{E})$. This latter map parallels the "embedding of the determinant" Eq. (5). Thus the only non-vanishing group $E_{\infty}^{j, k}(\mathscr{E})$ is the one with $j=k=0,\left\{\left[E_{1}^{0,0}(\mathscr{E}) / E_{1}^{0,1}(\mathscr{E})\right] / E_{1}^{2,3}(\mathscr{E})\right\}$, which is of rank 110-10-1. The normal bundle spectral sequence converges to $H^{q}\left(\mathscr{M}_{\mathrm{CY}}, \mathscr{E}\right)$ and guarantees that it vanishes except for $q=0$, when it is of dimension 99 .

By Corollary $4, b_{1,2}=\operatorname{dim} \mathfrak{D}\left(\mathscr{M}_{3}, \mathscr{W}_{3}\right)$, since $E_{1}^{1,1}\left(T_{\mathscr{W}}\right)=\emptyset$, and by Corollary 3 , $b_{1,1}=2$. This can also be seen from the exact sequence in the bottom row of the diagram (7), which falls apart into:

$$
\begin{array}{ccc}
0 \rightarrow H^{0}\left(\mathscr{M}_{3}, T_{\mathscr{W}}\right) & \rightarrow H^{0}\left(\mathscr{M}_{3}, \mathscr{E}\right) \rightarrow H^{1}\left(\mathscr{M}_{3}, T_{\mathscr{M}}\right) \rightarrow 0, \\
\operatorname{dim}=43 \quad \operatorname{dim}=99 & b_{1,2}
\end{array}
$$

and an even simpler part:

$$
\begin{gathered}
0 \rightarrow H^{2}\left(\mathscr{M}_{3}, T_{\mathscr{M}}\right) \rightarrow H^{2}\left(\mathscr{M}_{3}, T_{\mathscr{W}}\right) \rightarrow 0 . \\
b_{2,2} \quad \operatorname{dim}=2
\end{gathered}
$$

From the first sequence we conclude that $H^{1}\left(\mathscr{M}_{3}, T_{\mathscr{M}}\right)=H^{0}\left(\mathscr{M}_{3}, \mathscr{E}\right) / H^{0}\left(\mathscr{M}_{3}, T_{\mathscr{W}}\right)$ and has dimension 56 , while the second provides an isomorphism and therefore $b_{2,2}=b_{1,1}=2$.

\section{B. Examples Where PDM is Invalid}

Here we consider several examples for which PDM, as described in Sect. 3, would fail to detect some of the harmonic $(1,2)$-forms, and also provide some fake ones. Consider $\mathscr{M}_{4}$ :

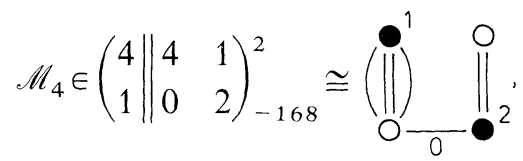

where the number below the decomposing leg denotes its index.

The ranks of $E_{1}^{0,0}(\mathscr{E}), E_{1}^{0,1}(\mathscr{E}), E_{1}^{0.0}\left(T_{\mathscr{W}}\right)$ and $E_{1}^{4,2}\left(T_{\mathscr{W}}\right)$ are easily computed much the same as in Appendix A, and are 85,2, 27 and 2 respectively. The first three of these correspond to the number of coefficients in the constraints, the two obvious rescalings of the two constraints and the coordinate transformations of $C P^{4} \times C P^{1}$ respectively. Corresponding to the decomposing 0 -leg, $E_{1}^{1,1}\left(T_{\mathscr{W}}\right)$ and $E_{1}^{1,1}(\mathscr{E})$ are also non-vanishing (since the dot-complement of the decomposing leg has $l_{d}-m_{d}$ =1). By Eq. (19), one obtains:

$$
E_{1}^{1,1}\left(T_{W^{\prime}}\right)=\left[H^{0}\left(C P_{1}^{4}, \xi_{1} \otimes \lambda_{1}^{-1}\right) \otimes H^{1}\left(C P_{2}^{1}, \lambda_{2}^{-2}\right)\right]
$$

where the second factor is, by duality, equivalent to $H^{0}\left(C P_{2}^{1}, \lambda_{2}^{0}\right)$ and has rank 1 . By Eq. (20), $E_{1}^{1,1}\left(T_{\mathscr{W}}\right)$ has rank 5 , but for sake of completeness, we present a computation of the rank of the first factor: consider the exact sequence:

$$
0 \rightarrow \lambda \rightarrow \mathbf{C}^{n+1} \rightarrow\left\{\mathbf{C}^{n+1} / \lambda\right\} \rightarrow 0,
$$

where $\lambda$ is the Hopf bundle of $C P^{n} C \mathbf{C}^{n+1}$. The dual of this sequence is:

$$
0 \rightarrow T_{C P^{n}} \otimes \lambda^{-1} \rightarrow \mathbf{C}^{n+1} \rightarrow \lambda^{-1} \rightarrow 0,
$$


since $T_{C P^{n}}=\lambda \otimes\left\{\mathbf{C}^{n+1} / \lambda\right\}^{*}[13]$. The last exact sequence induces the long exact sequence of cohomologies:

$$
0 \rightarrow H^{0}\left(T_{C P^{n}} \otimes \lambda^{-1}\right) \rightarrow H^{0}\left(\mathbf{C}^{n+1}\right) \rightarrow H^{0}\left(\lambda^{-1}\right) \rightarrow \ldots,
$$

where $H^{0}\left(\lambda^{-1}\right)$ vanishes; hence:

$$
H^{0}\left(T_{C P^{n}} \otimes \lambda^{-1}\right) \approx H^{0}\left(\mathbf{C}^{n+1}\right) \Rightarrow \operatorname{dim} H^{0}\left(T_{C P^{n}} \otimes \lambda^{-1}\right)=n+1 .
$$

Using this result, we obtain $\operatorname{rank} E_{1}^{1,1}\left(T_{\mathscr{W}}\right)=5$. This completes the computation of $E_{1}^{j, k}\left(T_{\mathscr{W}}\right)$ for $\mathscr{M}_{4}$ and by Theorem $2, E_{1}\left(T_{\mathscr{W}}\right)=E_{\infty}\left(T_{\mathscr{W}}\right)$. By Eq. (17), $\operatorname{dim} H^{0}\left(\mathscr{M}_{4}, T_{\mathscr{W}}\right)$ $=27+5=32$, and we see that $\mathfrak{D}\left(\mathscr{M}_{4}, \mathscr{W}_{4}\right)$ is ineffective as described in Sect. 5. One also obtains that $H^{1}\left(\mathscr{M}_{4}, T_{\mathscr{W}}\right)$ and $H^{3}\left(\mathscr{M}_{4}, T_{\mathscr{W}}\right)$ vanish, while $H^{2}\left(\mathscr{M}_{4}, T_{\mathscr{W}}\right)$ is twodimensional.

The non-vanishing element of $E_{1}^{j, k}(\mathscr{E})$ corresponding to the 0-leg is also the only remaining one and by Eq. (24) it is:

$$
E_{1}^{1,1}(\mathscr{E})=\left[H^{0}\left(C P_{1}^{4}, \lambda_{1}^{4-1}\right) \otimes H^{1}\left(C P_{2}^{1}, \lambda_{2}^{-2}\right)\right],
$$

the rank of which is $\left[\left(\begin{array}{l}7 \\ 3\end{array}\right) \cdot(1)\right]=35$. (This corresponds to $b=1$ and $A=\{2\}$.) Since there are no more non-vanishing groups in $E_{1}(\mathscr{E})$, and in particular on the 1-diagonal, by Corollary $3, b_{1,1}=2$. Using that $\chi_{E}=-168, b_{1.2}=86$. The same can be derived just from the spectral sequences by observing that the only nonvanishing differential is $d_{1}: E_{1}^{0,1}(\mathscr{E}) \rightarrow E_{1}^{0,0}(\mathscr{E})$ and it must be effective, yielding $E_{2}^{0,0}(\mathscr{E})=E_{1}^{0,0}(\mathscr{E}) / E_{1}^{0,1}(\mathscr{E}), E_{2}^{0,1}(\mathscr{E})=\emptyset$ and $E_{2}^{j, k}(\mathscr{E})=E_{1}^{j, k}(\mathscr{E})$ otherwise. Hence $E_{2}(\mathscr{E})$ $=E_{\infty}(\mathscr{E})$ and by Eq. (17): $\operatorname{dim} H^{0}\left(\mathscr{M}_{4}, \mathscr{E}\right)=83+35=118$ and $H^{q}\left(\mathscr{M}_{4}, \mathscr{E}\right)$ vanish for $q>0$. This shows that $\mathfrak{D}\left(\mathscr{M}_{4}, \mathscr{W}_{4}\right)$ is incomplete.

By Corollary 2:

$$
\begin{gathered}
0 \rightarrow H^{0}\left(\mathscr{M}_{4}, T_{\mathscr{W}}\right) \rightarrow H^{0}\left(\mathscr{M}_{4}, \mathscr{E}\right) \rightarrow H^{1}\left(\mathscr{M}_{4}, T_{\mathscr{M}}\right) \rightarrow 0, \\
\operatorname{dim}=32 \quad \operatorname{dim}=118 \quad b_{1,2} \\
0 \rightarrow H^{2}\left(\mathscr{M}_{4}, T_{\mathscr{M}}\right) \rightarrow H^{2}\left(\mathscr{M}_{4}, T_{\mathscr{W}}\right) \rightarrow 0, \\
b_{2,2} \quad \operatorname{dim}=2
\end{gathered}
$$

yielding $b_{1,2}=86$ and $b_{2,2}=2$ in accord with the straightforward computation $[4,10]$.

Next we consider:

$$
\left.\mathscr{M}_{5} \in\left(\begin{array}{l}
2 \\
2 \\
2 \\
1
\end{array}|| \begin{array}{llll}
1 & 1 & 1 & 0 \\
0 & 2 & 0 & 1 \\
0 & 0 & 1 & 2 \\
2 & 0 & 0 & 0
\end{array}\right)_{-56}^{4} \cong 0=\frac{1}{0}\right)_{0}^{1}
$$

since one can compute only $\chi_{E}=-56$ by straightforward methods and PDM leads to $b_{1,2}=23$, predicting thus $b_{1,1}=-5$, and is thus manifestly incorrect, while on the other side our Theorem also states that $\mathfrak{D}\left(\mathscr{M}_{5}, \mathscr{W}_{5}\right)$ is ineffective.

$E_{1}^{0,0}\left(T_{\mathscr{W}}\right), E_{1}^{6,4}\left(T_{\mathscr{W}}\right), E_{1}^{0,0}(\mathscr{E})$ and $E_{1}^{0,1}(\mathscr{E})$ are computed as before and yield their ranks to be $27,4,54$ and 4 respectively. By the standard argument, $E_{2}^{0,1}(\mathscr{E})$ vanishes and $E_{2}^{0,0}(\mathscr{E})=E_{1}^{0,0}(\mathscr{E}) / E_{1}^{0,1}(\mathscr{E})$ and has rank 50 .

Next we find the contributions corresponding to the 0-leg:

$$
E_{1}^{1,1}\left(T_{\mathscr{W}}\right)=\left[H^{0}\left(C P_{1}^{2}, \xi_{1} \otimes \lambda_{1}^{-1}\right) \otimes H^{0}\left(C P_{2}^{2}, \lambda_{2}^{0}\right) \otimes H^{0}\left(C P_{3}^{2}, \lambda_{3}^{0}\right) \otimes H^{1}\left(C P_{4}^{1}, \lambda_{4}^{-2}\right)\right],
$$


which is of rank 4 and is not accounted for by PDM, and by Theorem 2, $E_{1}\left(T_{\mathscr{W}}\right)$ $=E_{\infty}\left(T_{\mathscr{W}}\right)$. Collecting the groups on the diagonals (corresponding to the filtration in Eq. (16)) we obtain that $H^{q}\left(\mathscr{M}_{5}, T_{\mathscr{W}}\right)$ vanishes for $q=1,3$ and is of rank 30 and 4 for $q=0$ and 2 respectively.

Now we find the remaining non-vanishing groups $E_{1}^{j, k}(\mathscr{E})$. There are two nonvanishing terms in Eq. (22) and we list then with the subscripts and superscripts on the square brackets denoting the values of $b$ and $A$ in the direct sum:

$$
\begin{aligned}
E_{1}^{1,1}(\mathscr{E})= & {\left[H^{0}\left(C P_{1}^{2}, \lambda_{1}^{0}\right) \otimes H^{0}\left(C P_{2}^{2}, \lambda_{2}^{2}\right) \otimes H^{0}\left(C P_{3}^{2}, \lambda_{3}^{0}\right) \otimes H^{1}\left(C P_{4}^{1}, \lambda_{4}^{-2}\right)\right]_{b=2}^{A=\{1\}} } \\
& \oplus\left[H^{0}\left(C P_{1}^{2}, \lambda_{1}^{0}\right) \otimes H^{0}\left(C P_{2}^{2}, \lambda_{2}^{0}\right) \otimes H^{0}\left(C P_{3}^{2}, \lambda_{3}^{1}\right) \otimes H^{1}\left(C P_{4}^{1}, \lambda_{4}^{-2}\right)\right]_{b=3}^{A=\{1}
\end{aligned}
$$

with $\operatorname{rank} E_{1}^{1,1}(\mathscr{E})=\left[\left(\begin{array}{l}4 \\ 2\end{array}\right)\right]+\left[\left(\begin{array}{l}3 \\ 1\end{array}\right)\right]=9$. There is only one more non-vanishing group, for $|A|=2$ :

$$
\begin{aligned}
E_{1}^{2,2}(\mathscr{E})= & {\left[H^{0}\left(C P_{1}^{2}, \lambda_{1}^{0}\right) \otimes H^{0}\left(C P_{2}^{2}, \lambda_{2}^{1}\right) \otimes H^{0}\left(C P_{3}^{2}, \lambda_{3}^{0}\right)\right.} \\
& \left.\otimes H^{1}\left(C P_{4}^{1}, \lambda_{4}^{-2}\right)\right]_{b=2}^{A=\{3,4\}},
\end{aligned}
$$

with rank 3 . Therefore, $\supset\left(\mathscr{M}_{5}, \mathscr{W}_{5}\right)$ is incomplete and PDM fails to supply $9+3=12$ harmonic $(1,2)$-forms. Since there are no more non-vanishing groups in the normal bundle spectral sequence, all differentials must vanish. Thus, as before, $E_{2}(\mathscr{E})$ $=E_{\infty}(\mathscr{E})$ and we obtain that $H^{q}\left(\mathscr{M}_{5}, \mathscr{E}\right)$ vanishes for $q>0$ and is of rank 62 for $q=0$.

The long exact sequence in the bottom line of (7) now yields:

$$
\begin{gathered}
0 \rightarrow H^{0}\left(\mathscr{M}_{5}, T_{\mathscr{W}}\right) \rightarrow H^{0}\left(\mathscr{M}_{5}, \mathscr{E}\right) \rightarrow H^{1}\left(\mathscr{M}_{5}, T_{\mathscr{M}}\right) \rightarrow 0, \\
\operatorname{dim}=30 \quad \operatorname{dim}=62 \quad b_{1,2} \\
0 \rightarrow H^{2}\left(\mathscr{M}_{5}, T_{\mathscr{M}}\right) \rightarrow H^{2}\left(\mathscr{M}_{5}, T_{\mathscr{W}}\right) \rightarrow 0 \\
b_{2,2} \quad \operatorname{dim}=4
\end{gathered}
$$

Hence $H^{2,1}\left(\mathscr{M}_{5}\right)=H^{0}\left(\mathscr{M}_{5}, \mathscr{E}\right) / H^{0}\left(\mathscr{M}_{5}, T_{\mathscr{W}}\right)$ and $b_{1,2}=32, b_{1,1}=4$. (The latter two results could again have been obtained by Corollary 3 and using that $\chi_{E}=-56$.)

We give one more example where $\mathfrak{D}(\mathscr{M}, \mathscr{W})$ is both ineffective and incomplete, and straightforward methods only compute $\chi_{E}$ :

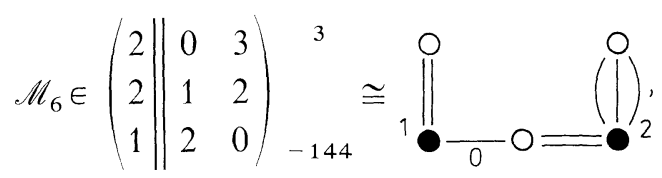

The spectral sequences are computed just as above and we obtain the following non-vanishing groups:

$$
\begin{array}{ccccccc}
\text { group } & E_{1}^{0,0}\left(T_{\mathscr{W}}\right) & E_{1}^{1,1}\left(T_{\mathscr{W}}\right) & E_{1}^{4,2}\left(T_{\mathscr{W}}\right) & E_{1}^{0,0}(\mathscr{E}) & E_{1}^{0,1}(\mathscr{E}) & E_{1}^{1,1}(\mathscr{E}) \\
\text { rank } & 19 & 3 & 3 & 69 & 2 & 30
\end{array}
$$

One readily concludes that again $E_{1}\left(T_{\mathscr{W}}\right)=E_{\infty}\left(T_{\mathscr{W}}\right)$, and that the only nonvanishing differential in the normal bundle spectral sequence is the $E_{1}^{0,1}(\mathscr{E})$ $\rightarrow E_{1}^{0,0}(\mathscr{E})$ one. The latter guarantees that $E_{2}^{0,0}(\mathscr{E})=\mathrm{E}_{1}^{0,0}(\mathscr{E}) / E_{1}^{0,1}(\mathscr{E})$, which is of rank 67 , and that $E_{2}^{0,1}(\mathscr{E})$ vanishes. Since $E_{2}^{j, k}(\mathscr{E})=E_{\infty}^{j, k}(\mathscr{E})$ for other $(j, k), E_{2}(\mathscr{E})$ $=E_{\infty}(\mathscr{E})$. 
Thus $H^{q}\left(\mathscr{M}_{6}, T_{\mathscr{W}}\right)$ vanishes for $q=1,3$ and is of rank 22 and 3 for $q=0$ and 2 respectively, while $H^{q}\left(\mathscr{M}_{6}, \mathscr{E}\right)$ vanishes for $q>0$ and is of rank 97 for $q=0$. $\mathfrak{D}\left(\mathscr{M}_{6}, \mathscr{W}_{6}\right)$ is both ineffective and incomplete.

Again, the long exact sequence in (7) now splits into:

$$
\begin{gathered}
0 \rightarrow H^{0}\left(\mathscr{M}_{6}, T_{\mathscr{W}}\right) \rightarrow H^{0}\left(\mathscr{M}_{6}, \mathscr{E}\right) \rightarrow H^{1}\left(\mathscr{M}_{6}, T_{\mathscr{M}}\right) \rightarrow 0, \\
\operatorname{dim}=22 \quad \operatorname{dim}=97 \quad b_{1,2} \\
0 \rightarrow H^{2}\left(\mathscr{M}_{6}, T_{\mathscr{M}}\right) \rightarrow H^{2}\left(\mathscr{M}_{6}, T_{\mathscr{W}}\right) \rightarrow 0 . \\
b_{2,2} \quad \operatorname{dim}=3
\end{gathered}
$$

Hence $H^{2,1}\left(\mathscr{M}_{6}\right)=H^{0}\left(\mathscr{M}_{6}, \mathscr{E}\right) / H^{0}\left(\mathscr{M}_{6}, T_{\mathscr{W}}\right)$ and $b_{1,2}=75, b_{1,1}=3$.

We now give an example where $E_{\infty}(\mathscr{E})$ cannot be computed straightforwardly, yet $b_{1,2}(\mathscr{M})$ and $b_{1,1}(\mathscr{M})$ can. We take:

$$
\mathscr{M}_{7}=\left(\begin{array}{l||lll}
3 \\
1 \\
1 \\
1
\end{array} \| \begin{array}{lll}
2 & 1 & 1 \\
1 & 1 & 0 \\
1 & 0 & 1 \\
0 & 0 & 2
\end{array}\right)=0
$$

Since there is no decomposing leg, $E_{1}\left(T_{\mathscr{W}}\right)=E_{\infty}\left(T_{\mathscr{W}}\right)$, where we readily compute the rank of $E_{1}^{0,0}\left(T_{\mathscr{W}}\right)$ to be 24 and $\operatorname{rank} E_{1}^{5,3}\left(T_{\mathscr{W}}\right)=4$. This yields $\operatorname{dim} H^{q}\left(\mathscr{M}, T_{\mathscr{M}}\right)=24,0$, 4 and 0 for $q=0,1,2$ and 3 respectively. Since $H^{1}\left(\mathscr{M}, T_{\mathscr{W}}\right)$ vanishes, Corollary 2 implies that $H^{1}\left(\mathscr{M}, T_{\mathscr{M}}\right)=H^{0}(\mathscr{M}, \mathscr{E}) / H^{0}\left(\mathscr{M}, T_{\mathscr{W}}\right)$.

$E_{1}^{0,0}(\mathscr{E})$ is readily computed to have rank 72 , which corresponds to the usual polynomial deformations of PDM. Further, Eq. (22) tells us that $E_{1}^{0,1}(\mathscr{E})$ has rank 11 (corresponding to the overall rescalings and embedding the second constraint into the first one). Equation (24) further yields a rank 8 contribution:

$$
\left[H^{0}\left(C P_{1}^{3}, \lambda_{1}^{2-1}\right) \otimes H^{0}\left(C P_{2}^{1}, \lambda_{2}^{1-0}\right) \otimes H^{0}\left(C P_{3}^{1}, \lambda_{3}^{1-1}\right) \otimes H^{1}\left(C P_{4}^{1}, \lambda_{4}^{-2}\right)\right]_{b=1}^{A=\{3\}}
$$

to $E_{1}^{1,1}(\mathscr{E})$, and a rank 1 contribution to $E_{1}^{1,2}(\mathscr{E})$ :

$$
\begin{gathered}
{\left[H^{0}\left(C P_{1}^{3}, \lambda_{1}^{2-1-1}\right) \otimes H^{0}\left(C P_{2}^{1}, \lambda_{2}^{1-1-0}\right) \otimes H^{0}\left(C P_{3}^{1}, \lambda_{3}^{1-0-1}\right)\right.} \\
\left.\otimes H^{1}\left(C P_{4}^{1}, \lambda_{4}^{-2}\right)\right]_{b=1}^{A=\{2,3\}} .
\end{gathered}
$$

These could not have been found by PDM as described in Sect. 3 .

Now it is manifest that $E_{2}^{0,0}(\mathscr{E})=E_{1}^{0,0}(\mathscr{E}) / E_{1}^{0,1}(\mathscr{E})$ has rank $72-11=61$ because of the action of $d_{1}$. However, it is not clear whether $d_{1}$ maps $E_{1}^{1,2}(\mathscr{E})$ into $E_{1}^{1,1}(\mathscr{E})$ reducing its rank to $8-1=7$, or $D_{1}$ vanishes here, $E_{2}^{1,2}(\mathscr{E})=E_{1}^{1,2}(\mathscr{E})$ and is mapped by $d_{2}$ into $E_{2}^{0,0}(\mathscr{E})$ reducing its rank to $61-1=60$. Thus $E_{\infty}(\mathscr{E})$ cannot be computed straightforwardly. However, by Corollary $3, b_{1,1}=4$ and using $\chi_{E}=-80$ one has that $b_{1,2}=44$.

The same could have been obtained from the spectral sequences alone, even though $E_{\infty}(\mathscr{E})$ is not computable, since whatever be the case $E_{1}^{1.2}(\mathscr{E})$ cannot survive to $E_{\infty}(\mathscr{E})$ and therefore must contribute a subtraction of 1 to the sum of ranks in Eq. (17). This assures the vanishing of $H^{q}(\mathscr{M}, \mathscr{E})$ except for $q=0$, when it has rank 68. 
As noted already, the long exact sequences in (7) again fall apart:

$$
\begin{gathered}
0 \rightarrow H^{0}\left(\mathscr{M}_{7}, T_{\mathscr{W}}\right) \rightarrow H^{0}\left(\mathscr{M}_{7}, \mathscr{E}\right) \rightarrow H^{1}\left(\mathscr{M}_{7}, T_{\mathscr{M}}\right) \rightarrow 0, \\
\operatorname{dim}=24 \quad \operatorname{dim}=68 \quad b_{1,2} \\
0 \rightarrow H^{2}\left(\mathscr{M}_{7}, T_{\mathscr{M}}\right) \rightarrow H^{2}\left(\mathscr{M}_{7}, T_{\mathscr{W}}\right) \rightarrow 0 . \\
b_{2,2} \quad \operatorname{dim}=4
\end{gathered}
$$

Hence $b_{1,2}=44, b_{2.2}=b_{1,1}=4$.

\section{Limitations on the Method}

Our next example is a $1 \mathrm{LD}$ configuration with a 1-leg, which illustrates the limitation of our, method in as much it does not compute $b_{1,2}$ and $b_{1.1}$ independently of the straightforward methods $[4,10]$ :

$$
\mathscr{M}_{8} \in\left(\begin{array}{l||ll}
3 \\
2
\end{array} \| \begin{array}{ll}
3 & 1 \\
0 & 3
\end{array}\right)^{8} \cong\left(\prod _ { - 5 4 } ^ { 1 } \underset { 1 } { O } \left(\left.\right|_{2} ^{O}\right.\right.
$$

The computation described above yields this time:

$$
\begin{array}{ccccccc}
\text { group } & E_{1}^{0,0}\left(T_{\mathscr{W}}\right) & E_{1}^{2,1}\left(T_{\mathscr{W}}\right) & E_{1}^{4,2}\left(T_{\mathscr{W}}\right) & E_{1}^{0,0}(\mathscr{E}) & E_{1}^{0,1}(\mathscr{E}) & E_{1}^{2,1}(\mathscr{E}) \\
\text { rank } & 23 & 4 & 2 & 60 & 2 & 10
\end{array}
$$

We again have $E_{1}\left(T_{\mathscr{W}}\right)=E_{\infty}\left(T_{\mathscr{W}}\right)$ and that the only non-trivial differential in the normal bundle spectral sequence is the $E_{1}^{0,1}(\mathscr{E}) \rightarrow E_{1}^{0,0}(\mathscr{E})$ one. Hence $E_{\infty}(\mathscr{E})$ $=E_{2}(\mathscr{E})$, which is the same as $E_{1}(\mathscr{E})$ except for $E_{2}^{0,1}(\mathscr{E})=\emptyset$ and $E_{2}^{0,0}(\mathscr{E})$ $=E_{1}^{0,0}(\mathscr{E}) / E_{1}^{0,1}(\mathscr{E})$, which has rank 58 .

This ensures that $\operatorname{dim} H^{q}\left(\mathscr{M}_{8}, T_{\mathscr{W}}\right)=23,4,2$ and 0 for $q=0,1,2$ and 3 respectively, as well as that $\operatorname{dim} H^{q}\left(\mathscr{M}_{8}, \mathscr{E}\right)=58,10,0$ and 0 for $q=0,1,2$ and 3 respectively. This time the long exact sequence in (7) does not split up but remains:

$$
\begin{aligned}
& 0 \longrightarrow H^{0}\left(\mathscr{M}_{8}, T_{\mathscr{W}}\right) \stackrel{j_{*}}{\longrightarrow} H^{0}\left(\mathscr{M}_{8}, \mathscr{E}\right) \stackrel{\delta}{\longrightarrow} H^{1}\left(\mathscr{M}_{8}, T_{\mathscr{M}}\right) \stackrel{i_{*}}{\longrightarrow} H^{1}\left(\mathscr{M}_{8}, T_{\mathscr{W}}\right) \\
& \operatorname{dim}=23 \quad \operatorname{dim}=58 \quad b_{1,2} \quad \operatorname{dim}=4 \\
& \stackrel{J_{*}}{\longrightarrow} H^{1}\left(\mathscr{M}_{8}, \mathscr{E}\right) \stackrel{\delta}{\longrightarrow} H^{2}\left(\mathscr{M}_{8}, T_{\mathscr{M}}\right) \stackrel{i_{*}}{\longrightarrow} H^{2}\left(\mathscr{M}_{8}, T_{\mathscr{W}}\right) \longrightarrow 0, \\
& \operatorname{dim}=10 \quad b_{1,1} \quad \operatorname{dim}=2
\end{aligned}
$$

and all it provides is that $35 \leqq b_{1,2} \leqq 39$ and $8 \leqq b_{1,1} \leqq 12$. Computing the Euler character fixes only their difference, thus one needs to obtain $b_{1,1}$ or $b_{1,2}$ by an independent method. Note that $\mathfrak{D}\left(\mathscr{C}_{8}, \mathscr{W}_{8}\right)$ is not ineffective since the configuration has no 0-leg, but we have no general enough criterion to detect incompleteness. Fortunately, the Lefschetz Hyperplane Theorem applies [10] and yields $b_{1,1}=8$, hence $b_{1,2}=35$. Since the lower bound of the spectral sequence computation of $b_{1,2}$ is what PDM predicts (corresponding to $i_{*}=0$ in dimension 1), PDM is valid in this case.

There are, however, 1LD configurations with a decomposing 1-leg where one can compute only the Euler character since the Lefschetz Hyperplane Theorem 
does not apply. A rather peculiar example of this kind is:

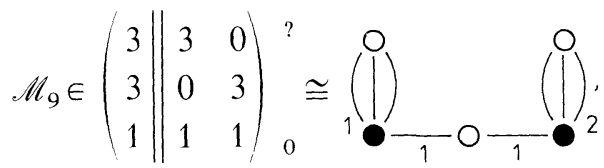

The fact that $\chi_{E}=0$ might give rise to the suspicion that $\mathscr{M}_{9}$ is just an embedding of $T^{2} \times K_{3}$ or $T^{2} \times T^{2} \times T^{2}$. This however is not true, and can be straightforwardly proven by the fact that the Hodge numbers of the latter manifolds do not agree with those of $\mathscr{M}_{9}$ computed by Theorem 1 .

Computing the groups in the other two spectral sequences we obtain:

$$
\begin{array}{ccccccc}
\text { group } & E_{1}^{0,0}\left(T_{\mathscr{W}}\right) & E_{1}^{2,1}\left(T_{\mathscr{W}}\right) & E_{1}^{4,2}\left(T_{\mathscr{W}}\right) & E_{1}^{0,0}(\mathscr{E}) & E_{1}^{0,1}(\mathscr{E}) & E_{1}^{2,1}(\mathscr{E}) \\
\text { rank } & 19 & 4 & 3 & 40 & 2 & 20
\end{array}
$$

Much the same as before: $E_{1}\left(T_{\mathscr{W}}\right)=E_{\propto}\left(T_{\mathscr{W}}\right)$; and $\mathcal{D}\left(\mathscr{M}_{9}, \mathscr{W}_{9}\right)$ is effective. Also, $E_{2}(\mathscr{E})$ $=E_{\infty}(\mathscr{E})$ and $E_{2}^{j . k}(\mathscr{E})=E_{1}^{j, k}(\mathscr{E})$ except for $(j, k)=(0,0)$ and $(0,1)$ when $E_{2}^{j, k}(\mathscr{E})$ is of rank 38 and 0 respectively. The long exact sequence in the diagram (7) lets us now to conclude that $19 \leqq b_{1,2} \leqq 23$ and $3 \leqq b_{1,1} \leqq 23$. Even after using that $\chi_{E}=2\left(b_{1.1}\right.$ $-b_{1.2}$ ) we have still only the bounds $19 \leqq b_{1,1}=b_{1.2} \leqq 23$.

Acknowledgements. It is a pleasure to thank M. Cvetič and S. Kalara for encouraging and helpful discussions. This work has been supported in part by the National Science Foundation and by the Self-Managing Community of Interest for Science of the Socialist Republic of Croatia.

\section{References}

1. Candelas, P., Horowitz, G.T., Strominger, A., Witten, E.: Vacuum configurations for superstrings. Nucl. Phys. B 258, 46 (1985)

2. Witten, E.: Symmetry breaking patterns in superstring models. Nucl. Phys. B 258, 75 (1985)

3. Schwarz, J.H.: Superstrings - a collection of reprints. Singapore: World Scientific 1985

4. Hübsch, T.: Calabi-Yau manifolds - motivations and constructions. Commun. Math. Phys. 108, 291-318 (1987)

5. Witten, E.: New issues in manifolds of $S U(3)$ holonomy. Nucl. Phys. B 268, 79 (1986)

6. Strominger, A., Witten, E.: New manifolds for superstring compactification. Commun. Math. Phys. 101, 341 (1985)

7. Hübsch, T.: Manifold compactification of superstrings. In: The proceedings of the summer workshop on high energy physics and cosmology. ICTP, Trieste, Italy 1986

8. Greene, B.R., Kirklin, K.H., Miron, P.J., Ross, G.G.: A three generation superstring model I. University of Oxford Report Ref. 26/86, A Superstring Inspired Standard Model. University of Oxford Report Print-86-1012

Goodman, M.W., Witten, W.: Global symmetries in four and higher dimensions. Nucl. Phys. B 271, 21 (1986)

Kalara, S., Mohapatra, R.N.: CP Violation and Yukawa couplings in superstring models. University of Minnesota Report UMN-TH-590-86, Phenomenology of a four generation superstring model. University of Maryland Report PP-87-123

Schimmrigk, R.: Superstring compactification with three generations. Institute for Theoretical Physics Report NSF-ITP-87-34

9. Kodaira, K.: Complex manifolds and deformation of complex structures. Berlin, Heidelberg, New York: Springer 1986 
10. Green, P., Hübsch, T.: Calabi-Yau manifolds as complete intersections in products of complex projective spaces. University of Maryland Report PP86-182. Commun. Math. Phys. 109, 99-108 (1987)

11. Bott, R.: Homogeneous vector bundles. Ann. Math. 66, 203 (1957)

12. Godement, R.: Theorie des faisceaux. Paris: Hermann 1958

Hirzebruch, F.: Topological methods in algebraic geometry. Berlin, Heidelberg, New York: Springer 1978

Griffiths, P., Harris, J.: Principles of algebraic geometry. New York: Wiley 1978

13. Szczarba, R.H.: On tangent bundle of fibre spaces and quotient spaces. Am. J. Math. 86, 685 (1964)

Hsiang, W.C., Szczarba, R.H.: On the tangent bundle of a Grassmann manifold. Am. J. Math. 86, 698 (1964)

14. Green, P., Hübsch, T.: Calabi-Yau hypersurfaces in products of semi-positive surfaces. University of Maryland Report PP-87-202

Communicated by A. Jaffe

Received March 27, 1987; in revised form May 29, 1987 